\title{
On the Stochastic Quantization of Field Theory
}

\author{
G. Jona-Lasinio ${ }^{1,2, \dagger}$ and P. K. Mitter ${ }^{2}$ \\ 1* Dipartimento di Fisica, Università di Roma "La Sapienza," Piazzale Aldo Moro 2, \\ I-00185 Roma, Italy \\ 2 Laboratoire de Physique Théorique et Hautes Energies ${ }^{\star \star}$, Université Pierre et Marie Curie, \\ Paris VI, F-75230 Paris Cedex 05, France
}

\begin{abstract}
We give a rigorous construction of a stochastic continuum $P(\phi)_{2}$ model in finite Euclidean space-time volume. It is obtained by a weak solution of a non-linear stochastic differential equation in a space of distributions. The resulting Markov process has continuous sample paths, and is ergodic with the finite volume Euclidean $P(\phi)_{2}$ measure as its unique invariant measure. The procedure may be called stochastic field quantization.
\end{abstract}

\section{Introduction}

Ever since the original work of Glauber [24], there has been much interest in stochastic statistical mechanical models. Such models have been rigorously studied by Holley, Stroock, Faris, Wick, and others [25-30]. The fundamental aim in these works is to obtain (and study properties of) Gibbs states of classical statistical mechanics as limiting distributions of stochastic processes. These processes are sometimes obtained as solutions of non-linear stochastic differential equations of the Langevin type (see later). Let $e^{t L}$ be the associated semi-group, and, starting from an initial state (probability measure) $\mu_{0}$, let $\mu_{t}$ be the evolved state under the action of the adjoint semigroup acting on the space of measures equipped with the weak * topology. If $\mu_{t} \rightarrow \mu$ in this topology, then $\mu$ is the unique equilibrium (invariant) measure. (Sometimes only a subsequence $\mu_{t_{K}}$ converges using weak commpactness criteria.) Let $\mu$ be an invariant measure. Then $\mu$ is a Gibbs state iff $e^{t L}$ is a selfadjoint contraction on $L^{2}(d \mu)$. If the invariant measure is unique, the process is ergodic.

In [18], Parisi and Wu proposed such a program for Euclidean quantum field theory. We may call this the method of stochastic quantization. This is natural because of the analogy between Euclidean quantum field theory and classical statistical mechanics. Euclidean quantum field theory is described by a probability

\footnotetext{
$\star$ Permanent address

$\star \star$ Laboratoire Associé 280 au CNRS

$\dagger$ Supported in part by GNSM and INFN
} 
measure which is formally Gibbsian and satisfies some important properties (reflection positivity, Euclidean invariance and some technical growth conditions), [1-3], which ensure the existence of its relativistic counterpart. However aside from Euclidean quantum mechanics (the so-called $P(\phi)_{1}$ models of which a stochastic version was constructed [19] by Faris and Jona-Lasinio) all non-trivial quantum field theories are plagued by ultraviolet (UV) divergences which necessitate renormalization. Under such singular circumstances it is not a priori obvious that the Parisi-Wu program can be rigorously pushed through. If it could, then the main interest of this program would be in its application to non-abelian gauge theories [18, 20-23].

In this paper we make a start on the rigorous non-perturbative analysis of this program. We consider the simplest non-trivial Euclidean quantum field theory which has, of course, ultraviolet divergence and hence necessitates renormalization, albeit of the simplest kind. This is the continuum $\left(\phi^{4}\right)_{2}$ model $[1,2,3]$ which we consider (as a first step) in a finite spacetime volume $\Lambda \subset R^{2}$, since we wish to concentrate on ultraviolet aspects. We consider $\Lambda$ to be a square, and the scalar fields $\phi$, with values in $R$, are given, for definiteness, Dirichlet boundary conditions. Such a theory is then specified by the finite measure [1-3] $\mu$ on $H_{-1}(\Lambda)$,

$$
\begin{gathered}
d \mu=d \mu_{c} e^{-\frac{\lambda}{4} \int d^{2} x: \phi^{4}:(x)}, \\
\underset{\text { formally }}{=} \int_{x \in \Lambda} d \phi(x) e^{-S(\phi)}, \\
S(\phi)=\frac{1}{2}(\phi,(-\Delta+1) \phi)_{L^{2}(\Lambda)}+\frac{\lambda}{4} \int_{\Lambda} d^{2} x: \phi^{4}:(x) .
\end{gathered}
$$

Here $H_{-1}(\Lambda)$ is a Sobolev space of distributions, $\mu_{c}$ is the Gaussian measure of mean 0 and covariance $C=(-\Delta+1)^{-1}$ with Dirichlet boundary conditions. : : denotes Wick ordering [1] with respect to $C$. Without Wick ordering the exponent in $(0.1)$ would not be a $\mu_{c}$ measurable random variable. The existence of $(0.1)$ is proved in $[1-3]$.

In this paper we will prove the existence of an ergodic, continuous, Markov process $\hat{\phi}_{t}$ with values in $H_{-1}(\Lambda)$ and having the measure $\mu$ of $(0.1)$ as its invariant measure. The process $\hat{\phi}_{t}$ is obtained as a weak solution of a generalized Langevin equation in the space of distributions $H_{-1}(\Lambda)$. Thus we have accomplished the stochastic quantization of finite volume $P(\phi)_{2}$ field theory.

In order to motivate the paper let us first consider a ferromagnetic statistical mechanical spin system (with values in $R$ ) in finite volume $\Lambda \subset Z^{2}$, with Gibbs distribution $\mu$ :

$$
\begin{gathered}
d \mu=\prod_{i \in \Lambda} d \phi(i) e^{-S(\phi)}, \\
S(\phi)=\frac{1}{2}(\phi,(-\Delta+1) \phi)+\frac{\lambda}{4} \sum_{i \in \Lambda} \phi^{4}(i),
\end{gathered}
$$

with $\lambda>0 . \Delta$ is the Lattice laplacian.

A symmetric diffusion process $\hat{\phi}_{t}$ which has (0.4) as its equilibrium measure will have a formal differential generator $L$ symmetric with respect to $L^{2}(d \mu)$. The most 
general $L^{2}(d \mu)$ symmetric second order differential operator $L$ is then of the form:

$$
L=\frac{1}{2} \sum_{i, j \in A} \tilde{K}(i, j) \frac{\delta^{2}}{\delta \phi(i) \delta \phi(j)}-\frac{1}{2} \sum_{i, j \in \Lambda} \tilde{K}(i, j) \frac{\delta S}{\delta \phi(j)} \frac{\delta}{\delta \phi(i)},
$$

where the operator $K: L^{2}(\Lambda) \rightarrow L^{2}(\Lambda)$,

$$
K f(i)=\sum_{j} \tilde{K}(i, j) f(j),
$$

is any bounded, positive, selfadjoint operator on $L^{2}(\Lambda)$. We shall restrict the choice of $K$ so that it commutes with $\Delta$.

In that case, formally, the dual semigroup (the Fokker-Planck semigroup) $e^{t L^{*}}$ leaves $\mu$ invariant, $e^{t L^{*}} \mu=\mu$, i.e. $\mu$ is an equilibrium distribution. The usual choice is $\tilde{K}(i, j)=\delta_{i j}$.

The diffusion process with $L$ [as in (0.6)] as its formal differential generator can be obtained by solving the Ito stochastic differential equation [also known as a (generalized) Langevin equation]

$$
\begin{aligned}
d \hat{\phi}_{t}(i) & =d W_{t}(i)-\frac{1}{2} \sum_{j} \tilde{K}(i, j) \frac{\delta S}{\delta \phi(j)}\left(\hat{\phi}_{t}\right) d t, \\
\hat{\phi}_{0}(i) & =\phi(i),
\end{aligned}
$$

and $W_{t}(i)$ is a Wiener process with covariance

$$
E\left(W_{t}(i) W_{s}(j)\right)=\tilde{K}(i, j) \min (t, s) .
$$

For finite lattice systems $\hat{\phi}_{t}$ can be rigorously constructed by standard methods. It can be shown to be ergodic, with $\mu$ of $(0.4)$ as its invariant measure, when the spectrum of the positive self-adjoint operator $K(-\Delta+1)^{-1}$ is bounded away from zero. The latter condition assures that the gaussian process $\phi_{t}$ [corresponding to the case $\lambda=0$ in (0.5)] has a mass gap. The existence of a solution of $(0.8)$ when $\Lambda \uparrow \mathbb{Z}^{2}$, and the existence of reversible invariant measures (Gibbs states) can also be proved [30].

We now turn to the continuum $P(\phi)_{2}$ model with $(0.1)-(0.3)$ replacing $(0.4)-(0.5)$. We write down the analogue of $(0.8)-(0.9)$ directly in the continuum. We choose for the operator $K$ [the analogue of $(0.7)]$ on $L^{2}\left(\Lambda, d^{2} x\right)$,

$$
K=C^{1-\varepsilon}=(-\Delta+1)^{-(1-\varepsilon)}
$$

with $\varepsilon \leqq 1$. Further restrictions will be imposed on $\varepsilon$ presently, for reasons to be explained below. Then the analogue of (0.8)-(0.9) reads, in operator notation,

$$
\begin{aligned}
d \hat{\phi}_{t} & =d W_{t}-\frac{1}{2}\left(C^{-\varepsilon} \hat{\phi}_{t}+\lambda C^{1-\varepsilon}: \hat{\phi}_{t}^{3}:\right) d t, \\
\hat{\phi}_{0} & =\phi,
\end{aligned}
$$

and $W_{t}$ is a Wiener process in $H_{-1}(\Lambda)$ with covariance

$$
E\left(W_{t}(f) W_{s}(g)\right)=\left(f, C^{1-\varepsilon} g\right) \min (t, s),
$$

where $f, g$ are test functions. 
Let $\phi_{t}$ be the unique solution of the linear stochastic differential equation in $H_{-1}(\Lambda)$,

$$
\begin{aligned}
d \phi_{t} & =d W_{t}-\frac{1}{2} C^{-\varepsilon} \phi_{t}, \\
\phi_{0} & =\phi
\end{aligned}
$$

$\phi_{t}$ is an Ornstein-Uhlenbeck (O.U.) process. Then $(0.11)$ can be written as an integral equation

$$
\hat{\phi}_{t}=\phi_{t}-\frac{\lambda}{2} \int_{0}^{t} d s e^{-\frac{1}{2}(t-s) C^{-\varepsilon}} C^{1-\varepsilon}: \hat{\phi}_{s}^{3}
$$

Note that : : is Wick ordering with respect to the covariance $C$.

Now for each starting point $\phi$, and time $t>0$, the transition probability of the O.U. process is a Gaussian measure $p_{t}\left(\phi, d \phi^{\prime}\right)$ on $H_{-1}(\Lambda)$. In order that $(0.14)$ be free of UV divergence it is necessary and sufficient that the Gaussian measures $p_{t}(\phi, \cdot)$ and $\mu_{\mathrm{c}}$ be equivalent. This imposes the condition $\varepsilon>0$, strictly.

Now the standard method of solving a non-linear equation like $(0.11,0.14)$ (Picard's method or contraction mapping) cannot be applied here since the perturbing drift [the non-linear term in $(0.11)$ ] has no continuity properties. $: \phi^{3}:(f)$ exists only in $L^{p}\left(d \mu_{c}\right), 1 \leqq p<\infty$. Instead we may attempt to construct a weak solution. ${ }^{1}$

The semi-group corresponding formally to the evolution governed by $(0.11)$ can be written as:

$$
\left(e^{t L} f\right)(\phi)=E_{\phi_{0}=\phi}^{(W)}\left(f\left(\phi_{t}\right) e^{-\frac{\lambda}{2} \int_{0}^{t}\left(: \phi_{s}^{3}:, d W_{s}\right)-\frac{\lambda^{2}}{8} \int_{0}^{t} d s\left(: \phi_{s}^{3}:, C^{1-\varepsilon}: \phi_{s}^{3}:\right)}\right),
$$

where $f$ is a bounded $\mu_{c}$ measurable function and $\phi_{t}$ is the O.U. process satisfying $(0.13)$, and is considered as a functional of the Wiener process $W_{t}$, and $E^{(W)}$ is Wiener expectation. For $(0.15)$ to be well defined, it is necessary that the exponent is a well defined random variable. This imposes the further restriction $\varepsilon<1$ strictly.

In the range $0<\varepsilon<1$ one shows straightforwardly that

$$
E_{\phi}^{(W)}\left(e^{\xi_{t}}\right) \leqq 1
$$

where $\xi_{t}$ is the exponent in $(0.15)$. Thus $e^{t L}$ is a contraction on $L^{\infty}\left(d \mu_{c}\right)$. However in order to construct a weak solution we must have

$$
E_{\phi}^{(W)}\left(e^{\xi_{t}}\right)=1
$$

To prove this we first obtain an Ito formula which permits us to rewrite $(0.15)$ in Feynman-Kac form. Exploiting the fact that the perturbing drift in (0.11) is a gradient, one obtains

$$
\int_{0}^{t}\left(: \phi_{s}^{3}:, d W_{s}\right)=\frac{1}{4} \int_{\Lambda}: \phi_{t}^{4}: d^{2} x-\frac{1}{4} \int_{\Lambda}: \phi_{0}^{4}: d^{2} x+\frac{1}{2} \int_{0}^{t} d s:\left(\phi_{s}^{3}, C^{-\varepsilon} \phi_{s}\right): .
$$

1 By a weak solution of $(0.14)$ we mean a Markov family of measures $\hat{P}_{\phi}$ on path space, such that $\hat{\phi}_{t}$ minus the second term in (0.14) has the same distributions as that of $\phi_{t}$ 
In order that this formula makes sense in $L^{2}\left(d P^{(W)}, \Omega\right)$, where $P^{(W)}$ is Wiener measure and $\Omega=C^{0}\left([0, \infty), H_{-1}(\Lambda)\right)$ is path space, we must impose the further restriction: $0<\varepsilon<\frac{1}{2}$, (otherwise the last term in (0.18) is not a random variable). Equation (0.18) enables us to eliminate the stochastic integral (0.15). Under the further and final restriction:

$$
0<\varepsilon<\frac{1}{10}
$$

(which is not necessarily optimal), we show, using estimates of the type encountered in constructive field theory $[1-3]$, that $e^{t L}$ is a strongly continuous self-adjoint contractive semi-group on $L^{2}(d \mu)$ with 1 as its unique ground state,

$$
e^{t L} 1=1
$$

which implies (0.17). The Markov process $\hat{\phi}_{t}$ is constructed, in a standard way, using the transition probabilities given by $e^{t L}$. There exists a continuous version of the process corresponding to a Markov family of measures $\hat{P}_{\phi}$ supported on $\Omega=C^{0}\left([0, t), H_{-1}(\Lambda)\right)$. It gives a weak solution of $(0.11)$ or $(0.14)$ in the sense that the process

$$
\hat{Z}_{t}=\hat{\phi}_{t}+\frac{\lambda}{2} \int_{0}^{t} d s e^{-\frac{1}{2}(t-s) C^{-\varepsilon}} C^{1-\varepsilon}: \hat{\phi}_{s}^{3}:
$$

has the same joint probability distributions (with respect to $\hat{P}_{\phi}$ ) as those of the O.U. process $\phi_{t}$. Thus $\hat{Z}_{t}$ can be identified as an O.U. process which is however not necessarily measurable with respect to $\phi_{t}$.

The Markov process $\hat{\phi}_{t}$ is ergodic, and also mixing (because of the existence of a mass gap). This ensures that

$$
\lim _{t \rightarrow \infty} E_{\phi}\left(\hat{\phi}_{t}\left(f_{1}\right) \ldots \hat{\phi}_{t}\left(f_{n}\right)\right)=\frac{1}{Z} \int d \mu \phi\left(f_{1}\right) \ldots \phi\left(f_{n}\right), \quad \mu \text { a.e. }
$$

where the $f_{i}$ are test functions and $Z=\int d \mu$.

The rest of this paper gives the technical details of the above outline. We have gone into some pedagogic details as the subject is of interest to both physicists and mathematicians.

\section{Preliminary: The $\left(\phi^{4}\right)_{2}$ Finite Volume Euclidean Measure}

Let $\Lambda \subset R^{2}$ be a square with Dirichlet boundary conditions. The Laplacian

$$
\Delta=\frac{\partial^{2}}{\partial x_{1}^{2}}+\frac{\partial^{2}}{\partial x_{2}^{2}}
$$

with the above boundary conditions is a self-adjoint operator on $L^{2}(\Lambda) . H_{1}(\Lambda)$ is the Sobolev Hilbert space of functions with norm $\|\cdot\|_{1}$,

$$
\|f\|_{1}^{2}=\int_{\Lambda} d^{2} x\left|(-\Delta+1)^{1 / 2} f(x)\right|^{2},
$$


obtained by completing $C^{\infty}(\Lambda)$ functions in this norm. $H_{-1}(\Lambda) \equiv E$ is the SobolevHilbert space of distributions with norm $\|\cdot\|_{-1}$,

$$
\|\phi\|_{-1}^{2}=\int_{A} d^{2} x\left|(-\Delta+1)^{-1 / 2} \phi(x)\right|^{2} .
$$

Then the injection:

$$
i: H_{1}(\Lambda) \hookrightarrow H_{-1}(\Lambda) \equiv E
$$

is Hilbert-Schmidt. A theorem of Sazonov, Minlos [11] and Gross [8,9] assures us that there exists a Gaussian probability measure on $E$ with mean 0 and covariance $C$, denoted $\mu_{c}$,

$$
\int_{E} d \mu_{c} \phi(f) \phi(g)=(f, C g),
$$

where $f, g \in C^{\infty}(\Lambda)$ are test functions, and

$$
C=(-\Delta+1)^{-1} \text {. }
$$

: : denotes Wick ordering [1-3] with respect to covariance $C$. It is defined recursively via

$$
\begin{aligned}
& \phi\left(f_{n}\right): \phi\left(f_{1}\right) \ldots \phi\left(f_{n-1}\right): \\
& \quad=: \phi\left(f_{1}\right) \ldots \phi\left(f_{n-1}\right) \phi\left(f_{n}\right):+\sum_{j=1}^{n-1}\left(f_{n}, C f_{j}\right): \phi\left(f_{1}\right) \ldots \phi\left(f_{j}\right) \ldots \phi\left(f_{n-1}\right):
\end{aligned}
$$

where $\checkmark$ means omission. Wick ordering gives a symmetric function. Let $\left\{\mathrm{e}_{\mathrm{n}}, \lambda_{n}\right\}$ be the spectral basis of $(-\Delta+1)$ on $L^{2}(\Delta)$.

Because we are in two dimensions, $\sum_{n=1}^{\infty} \frac{1}{\lambda_{n}}$ is logarithmically divergent and

$$
\sum_{n=1}^{\infty} \lambda_{n}^{-1-\delta}<\infty, \quad \delta>0
$$

This will be used repeatedly. For any $\phi \in E$,

$$
\phi=\sum_{n=1}^{\infty}\left(\phi, e_{n}\right) e_{n}=\sum_{n=1}^{\infty} \phi_{n} e_{n}
$$

and

$$
\phi^{(N)}=\sum_{n=1}^{N} \phi_{n} e_{n}
$$

is the $N$-finite mode approximation. We can define recursively $: \phi^{(N)}$ : by

$$
\begin{aligned}
\phi^{(N)}(x):\left(\phi^{(N)}(x)\right)^{n}: & =:\left(\phi^{(N)}(x)\right)^{n+1}:+n C^{(N)}(x):\left(\phi^{(N)}(x)\right)^{n-1}:, \\
C^{(N)}(x) & =\int d \mu_{c}\left(\phi^{(N)}(x)\right)^{2} .
\end{aligned}
$$

Then we have [1-3], the following facts:

$$
\begin{gathered}
:\left(\phi^{(N)}\right)^{n}:(f) \underset{N \rightarrow \infty}{\longrightarrow}: \phi^{n}(f) \text { in } L^{p}\left(d \mu_{c}, E\right), \quad 1 \leqq p<\infty, \\
e^{-\lambda \int_{\Lambda} d^{2} x: \phi^{4}:(x)} \in L^{1}\left(d \mu_{c}, E\right) .
\end{gathered}
$$


This leads to the definition of the finite volume Euclidean $\left(\phi^{4}\right)_{2}$ measure [1-3]

$$
d \mu=d \mu_{c} e^{-\frac{\lambda}{4} \iint_{A}^{4}:(x) d^{2} x} .
$$

Because of Nelson's estimate (1.11), (1.12) is a well defined finite measure supported on $H_{-1}(\Lambda)$.

In the following we also need the space of vector valued ( $E$-valued) functions on $E$, denoted $V_{E}$. The corresponding $L^{p}$ spaces, $L^{p}\left(d \mu_{c}, V_{E}\right)$ are defined with the norm:

$$
\|\mathscr{V}\|_{p}=\left(\int_{E} d \mu_{c}(\phi)\|\mathscr{V}(\phi)\|_{-1}^{p}\right)^{1 / p}
$$

It is easy to check : $\phi^{n}$ : belongs to $L^{P}\left(d \mu_{c}, V_{E}\right), 1 \leqq p$.

\section{Ornstein-Uhlenbeck Process Associated to the Euclidean Free Field, and Some Ito Functionals Thereof}

Throughout this section we hold $\varepsilon$ in the range $0<\varepsilon<\frac{1}{2}$.

Let $t$ be a positive real number, and define:

$$
C_{t}=t C^{1-\varepsilon}=t(-\Delta+1)^{-(1-\varepsilon)} .
$$

Let $\mu_{C_{t}}$ be a family of Gaussian measures supported on

$$
E=H_{-1}(\Lambda)
$$

of mean 0 and covariance $C_{t}$ obtained by the Sazonov-Minlos-Gross construction of the previous section.

For any $t>0, \phi \in E$, we define a family of probability measures $p_{t}^{(W)}(\phi, \cdot)$ on $E$ by the formula:

$$
p_{t}^{(W)}(\phi, B)=\mu_{C_{t}}(B-\phi),
$$

where $B$ is a Borel set in $E$.

The Wiener process $W_{t}$ in $E$ is defined by giving a family of probability measures $P_{\phi}^{(W)}$ with $\phi \in E$ on the path space $(\Omega, \mathbb{B}(\Omega))$, where $\Omega=C^{0}([0, \infty), E)$ with $\mathbb{B}$ its Borel algebra, satisfying

$$
P_{\phi}^{(W)}\left\{W_{t} \in B\right\}=p_{t}^{(W)}(\phi, B)
$$

the transition probability,

(ii) for $s<t$,

$$
\begin{gathered}
P_{\phi}^{(W)}\left\{\left(W_{t}-W_{s}\right) \in B\right\}=p_{t-s}^{(W)}(0, B), \\
P_{\phi}^{(W)}\left\{W_{s} \in A,\left(W_{t}-W_{s}\right) \in B\right\}=p_{s}^{(W)}(\phi, A) p_{t-s}^{(W)}(0, B) .
\end{gathered}
$$

(i), (ii), and (iii) guarantee that $P_{\phi}^{(W)}$ is a Markov process supported on $C^{0}$ $([0, \infty), E)$. The covariance is given by:

$$
E_{0}^{(W)}\left(W_{t}(f) W_{s}(g)\right)=\left(f, C^{1-\varepsilon} g\right) \min (t, s),
$$

where $E_{\phi}^{(W)}$ is integration with respect to $d P_{\phi}^{(W)}$ and $f, g$ are test functions. 
The Wiener process $W_{t}$ plays only an auxiliary role in the following. Our basic reference process will be an Ornstein-Uhlenbeck (O.U.) process $\phi_{t} \in E$, obtained as the unique solution of the linear stochastic differential equation,

$$
d \phi_{t}=d W_{t}-\frac{1}{2} C^{-\varepsilon} \phi_{t} d t
$$

The unique solution is

$$
\phi_{t}=e^{-\frac{t}{2} C^{-\varepsilon}} \phi+\int_{0}^{t} e^{-\frac{1}{2}(t-s) C^{-\varepsilon}} d W_{s},
$$

which describes $\phi_{t}$ as an explicit functional $\phi_{t}(W)$ of the Wiener process.

Remark on the Stochastic Integral in (2.7). The stochastic integral of the type

$$
I_{t}=\int_{0}^{t} e^{-\frac{1}{2}(t-s) C^{-\varepsilon}} d W_{s}
$$

encountered in (2.7) is a continuous square integrable martingale in $E$, with zero Wiener expectation, and the martingale property is with respect to $\sigma$-algebras $B_{t}$ engendered by $W_{s}, \forall s \leqq t$.

To see this we introduce a finite dimensional approximation $W_{s}^{(N)}$ to $W_{s}$ :

$$
W_{s}^{(N)}=\sum_{n=1}^{N} \lambda_{n}^{-\frac{1}{2}(1-\varepsilon)} \beta_{s}^{(n)} e_{n}
$$

where $\left\{\lambda_{n}^{-1}, e_{n}\right\}$ is an eigenbasis of $C$ on $L^{2}(\Lambda)$, and $\beta_{s}^{(n)}$ is normalized Brownian motion on the line:

$$
E\left(\beta_{s}^{(n)} \beta_{t}^{(m)}\right)=\delta_{n m} \min (t, s)
$$

Then

$$
I_{t}^{(N)}=\int_{0}^{t} \sum_{n=1}^{N} e^{-\frac{1}{2}(t-s) \lambda_{n}^{\varepsilon}} \lambda_{n}^{-\frac{1}{2}(1-\varepsilon)} d \beta_{s}^{(n)} e_{n}
$$

as an Ito Stochastic Integral in $R^{N},[13-16]$, is a continuous square integrable martingale. We have

$$
E_{0}^{(W)}\left(\left\|I_{t}^{(N+p)}-I_{t}^{(N)}\right\|_{E}^{2}\right)=\sum_{n=N}^{N+p} \frac{1}{\lambda_{n}^{2}}\left(1-e^{-\lambda_{n}^{\varepsilon} t}\right) \underset{N \rightarrow \infty}{\longrightarrow} 0
$$

since $\sum_{n=1}^{\infty} \lambda_{n}^{-2}<\infty$. Hence $\left\{I_{t}^{(N)}\right\}$ is a mean square convergent sequence, and its limit defines $I_{t}(f)$. To extract a continuous version we have by the martingale inequality [13],

$$
\begin{aligned}
P_{0}^{(W)} & \left\{\sup _{0 \leqq t \leqq T}\left\|I_{t}^{(N+p)}-I_{t}^{(N)}\right\|_{E}>\delta\right\} \leqq \delta^{-2} E_{0}^{(W)}\left(\left\|I_{T}^{(N+p)}-I_{T}^{(N)}\right\|_{E}^{2}\right) \\
\quad & \delta^{-2} \sum_{n=N}^{N+p} \frac{1}{\lambda_{n}^{2}}\left(1-e^{-t \lambda_{n}^{\varepsilon}}\right) \underset{N \rightarrow \infty}{\longrightarrow} 0 .
\end{aligned}
$$

By a standard application of the Borel-Cantelli lemma [13] we can extract from $I_{t}^{(N)}$ an a.e. convergent (uniformly in $t$ ) subsequence. Its limit defines the continuous version of $I_{t}$. 
The O.U. process $\phi_{t}^{(W)}$ starting at $\phi$ given by (2.7) is a continuous Markov process, which is not equivalent to the Wiener process $W_{t}$ (starting at $\phi$ ). $P_{\phi}$ is the probability measure on $C^{0}([0, \infty), E) \equiv \Omega$ induced by the process. $E_{\phi}$ is the corresponding expectation.

The transition probabilities $p_{t}(\phi, \cdot)$

$$
p_{t}(\phi, B)=P_{\phi}\left(\phi_{t} \in B\right)=P_{\phi}^{(W)}\left(W: \phi_{c}(W) \in B\right),
$$

with $B$ a Borel set in $E$ can be obtained as follows.

Let

$$
\phi=\sum_{n=1}^{\infty}\left(\phi, e_{n}\right) e_{n}=\sum_{n=1}^{\infty} \phi_{n} e_{n}
$$

with $\left\{\lambda_{n}^{-1}, e_{n}\right\}$ the eigenbasis of $C$ on $L^{2}(\Lambda)$. Then in components (2.7) reads:

$$
\phi_{t}^{(n)}=e^{-\frac{1}{2} \lambda_{n}^{\varepsilon}} \phi^{(n)}+\int_{0}^{t} e^{-\frac{1}{2}(t-s) \lambda_{n}^{\varepsilon}} \lambda_{n}^{-\frac{1}{2}(1-\varepsilon)} d \beta_{s}^{(n)},
$$

where $\beta_{s}^{(n)}$ is as in (2.9), (2.10).

The stochastic integral on the right-hand-side of (2.16) is also Brownian motion with time change [13], $t \rightarrow \tau_{n}(t)=\frac{1}{\lambda_{n}}\left(1-e^{-\lambda_{n}^{\varepsilon} t}\right)$. Noting also the translation by $e^{-(t / 2) \lambda_{n}^{\varepsilon}} \phi^{(n)}$, the component process $\phi_{t}^{(n)}$ has transition probabilities

$$
p_{\tau_{n}(t)}^{(W)}\left(e^{-\frac{1}{2} t \lambda_{n}^{\varepsilon}} \phi^{(n)}, \cdot\right)
$$

where $p_{t}^{(W)}(x, \cdot)$ is the standard Wiener transition probability in $R^{1}$.

It follows that the O.U. process $\phi_{t}$ of (2.7) has transition probabilities $p_{t}(\phi, \cdot)$ given by:

$$
p_{t}(\phi, B)=\mu_{\tilde{C}_{t}}\left(B-e^{-\frac{t}{2} c^{-\varepsilon}} \phi\right)
$$

where

$$
\widetilde{C}_{t}=\left(1-e^{-t C^{-\varepsilon}}\right) C,
$$

and $B$ is a Borel set in $E$.

Note that

$$
p_{t}(\phi, \cdot) \underset{t \rightarrow \infty}{\longrightarrow} \mu_{c}(\cdot)
$$

in the sense of weak convergence of measures. It follows that the O.U. process is ergodic with $\mu_{c}$ as its unique invariant measure.

If we use the Ito calculus, appropriate to $W_{t}$,

$$
\begin{gathered}
E^{(W)}\left(d W_{s}(f)\right)=0, \\
E^{(W)}\left(d W_{s}(f) d W_{s}(g)\right)=d s\left(f, C^{1-\varepsilon} g\right),
\end{gathered}
$$


where $f, g$ are test functions. We have directly from (2.7)

$$
\begin{aligned}
& E_{\phi}\left(\phi_{t}(f) \phi_{t}(g)\right)=E^{(W)}\left(\phi_{t}(W)(f) \phi_{t}(W)(g)\right) \\
& \quad=\phi\left(e^{-t / 2 C^{-\varepsilon}} f\right) \phi\left(e^{-t / 2 C^{-\varepsilon}} g\right)+\left(f,\left(1-e^{-t C^{-\varepsilon}}\right) C g\right) \underset{t \rightarrow \infty}{\longrightarrow}(f, C g) \\
& \quad=\int_{E} d \mu_{c} \phi(f) \phi(g) .
\end{aligned}
$$

Remark. The above construction of $P_{\phi}$, our equivalently of $\phi_{t}(W)$, may be called the "stochastic quantization" of the Euclidean free field in the sense of [18]. It should be remarked however that the white noise in [18] has unit covariance, whereas our white noise ( $\dot{W}_{t}$ formally) has covariance $C^{1-\varepsilon}$.

The drifts differ correspondingly. The advantage of our procedure will be the easy mathematical control of the stochastic quantization of the interacting $\left(\phi^{4}\right)_{2}$ Euclidean theory in the subsequent sections.

\section{The O.U. Semigroup $e^{t L_{0}}$ and Its Properties}

The continuous Markov process $\phi_{t}$ gives rise to a semigroup $e^{t L_{0}}$, the O.U. semigroup

$$
\left(e^{t L_{0}} f\right)(\phi)=E_{\phi}\left(f\left(\phi_{t}\right)\right)=\int_{E} p_{t}\left(\phi, d \phi^{\prime}\right) f\left(\phi^{\prime}\right),
$$

where $f: E \rightarrow R$ is a bounded measurable function (to begin with). The following proposition summarises well known properties of the O.U. semigroup, which suffice to control the $\left(\phi^{4}\right)_{2}$ stochastic quantization.

Proposition. The O.U. semigroup $e^{t L_{0}}$ satisfies

(i) $e^{t L_{0}}$ is positivity preserving and $e^{t L_{0}} 1=1$,

(ii) $e^{t L_{0}}$ is a contraction on all $L^{p}\left(d \mu_{c}\right), 1 \leqq p<\infty$,

(iii) $e^{t L_{0}}$ is a strongly continuous, contractive, self-adjoint semigroup on $L^{2}\left(d \mu_{c}\right)$,

(iv) $e^{t L_{0}}$ is hypercontractive: $\exists T>0$ such that for $t>T$

$$
\left\|e^{t L_{0}} f\right\|_{L^{4}\left(d \mu_{c}\right)} \leqq\|f\|_{L^{2}\left(d \mu_{c}\right)}
$$

(v) 1 is the unique ground state. $e^{t L_{0}}$ is positivity improving, i.e. if $f, g \geqq 0$ a.e. are non zero vectors in $L^{2}\left(d \mu_{c}\right)$, then $\left(f, e^{t L_{0}} g\right)>0$.

Note that (i) follows from $(2.23)$ and that $p_{t}(\phi, \cdot)$ is a probability measure (2.18). (ii) follows from (i) and the Markov property, see [12, Chap. XIII]. (iii) follows from (ii), symmetry of the transition probabilities and stochastic continuity: $p_{t}(\phi, B)$ is continuous in $t$, a.e. in $\phi$. (iv) was first isolated in the context of constructive field theory [2-7]. (v) follows from ergodicity, (i) [and selfadjointness in $L^{2}\left(d \mu_{c}\right)$ ], see [2].

\section{An Ito Formula}

We shall now derive an Ito formula which will play a key role in the subsequent sections.

By property (iii) of the previous proposition, if $L_{0}$ is the infinitesimal generator, then $-L_{0}$ is a non-negative selfadjoint operator. It can be realized as a second order differential operator on the subspace of twice differentiable cylindrical 
functions which with their derivatives are also in $L^{2}\left(d \mu_{c}\right)$. This subspace is dense in $\mathscr{D}\left(L_{0}\right)$.

Let

$$
\phi^{(N)}=\sum_{i=1}^{N} e_{i}\left(\phi, e_{i}\right)=\sum_{i=1}^{N} \phi_{i} e_{i},
$$

the (finite) mode expansion of (2.15).

Then a cylindrical function is of the form

$$
F\left(\phi^{(N)}\right)=F\left(\phi_{1}, \ldots, \phi_{N}\right),
$$

and on a twice differentiable cylindrical function which, with its derivatives, is in $L^{2}\left(d \mu_{c}\right)$

$$
L_{0} F\left(\phi^{(N)}\right)=\frac{1}{2} \sum_{n=1}^{N}\left(\lambda_{n}^{-(1-\varepsilon)} \frac{\partial^{2}}{\partial \phi_{n}^{2}}-\lambda_{n}^{\varepsilon} \phi_{n} \frac{\partial}{\partial \phi_{n}}\right) F\left(\phi^{(N)}\right)
$$

Define

$$
\begin{aligned}
F(\phi) & =\frac{1}{4} \int_{A} d^{2} x: \phi^{4}:(x), \\
H(\phi) & =\left(: \phi^{3}:, C^{1-\varepsilon}: \phi^{3}:\right), \\
G(\phi) & =:\left(\phi^{3}, C^{-\varepsilon} \phi\right):,
\end{aligned}
$$

and we recall that we restrict $\varepsilon$ to the range $0<\varepsilon<\frac{1}{2}$. Then $F, G, H \in L^{p}\left(d \mu_{c}\right)$, $1 \leqq p<\infty$, and $F\left(\phi^{(N)}\right), G\left(\phi^{(N)}\right), H\left(\phi^{(N)}\right)$ converge respectively to $F(\phi), G(\phi), H(\phi)$ in $L^{p}\left(d \mu_{c}\right)$. By explicit calculation,

$$
L_{0} F\left(\phi^{(N)}\right)=-\frac{1}{2} G\left(\phi^{(N)}\right) .
$$

We now note the following: the transition probabilities $p_{t}(\phi, \cdot)$ of the O.U. process $\phi_{t}$ given by (2.18) are absolutely continuous with respect to $\mu_{c}\left(d \phi^{\prime}\right)$ for each $\phi \in E$, $t>0$ provided $\varepsilon>0$. The Radon-Nikodym derivative

$$
\varrho_{t, \phi}\left(\phi^{\prime}\right)=\frac{p_{t}\left(\phi, d \phi^{\prime}\right)}{\mu_{c}\left(d \phi^{\prime}\right)}
$$

is in $L^{2}\left(d \mu_{c}\right)$. Indeed by a straightforward calculation

$$
\begin{aligned}
a(t, \phi)^{2} & \equiv \int\left|\varrho_{t, \phi}\left(\phi^{\prime}\right)\right|^{2} d \mu_{c}\left(\phi^{\prime}\right) \\
& =\left[\operatorname{det}\left(I-e^{-2 t C^{-\varepsilon}}\right)\right]^{-1 / 2} \exp \left\{\left(\phi, C^{-1} e^{-t C^{-\varepsilon}}\left(1+e^{-t C^{-\varepsilon}}\right)^{-1} \phi\right)\right\},
\end{aligned}
$$

which is well defined for $\varepsilon>0$, because then $e^{-2 t C^{-\varepsilon}}$ is of trace class.

Let $h \in L^{2 p}\left(d \mu_{c}\right)$. Then,

$$
\begin{aligned}
E_{\phi}\left(\left|h\left(\phi_{t}\right)\right|^{p}\right) & =\int p_{t}\left(\phi, d \phi^{\prime}\right)\left|h\left(\phi^{\prime}\right)\right|^{p} \\
& =\int \varrho_{t, \phi}\left(\phi^{\prime}\right)\left|h\left(\phi^{\prime}\right)\right|^{p} d \mu_{c}\left(\phi^{\prime}\right) \leqq a(r, \phi)\|h\|_{L^{2 p}\left(d \mu_{c}\right)}^{p} .
\end{aligned}
$$

As a consequence, $h \in L^{2 p}\left(d \mu_{c}\right), 1 \leqq p<\infty \Rightarrow h\left(\phi_{t}\right) \in L^{p}\left(d P_{\phi}, \Omega\right), 1 \leqq p<\infty$.

Finally let $h(\phi) \in L^{2 p}\left(d \mu_{c}\right)$ be obtained as the convergent limit of $h\left(\phi^{(N)}\right), N \rightarrow \infty$, where $h\left(\phi^{(N)}\right)$ is a continuous function of $\phi^{(N)}$. Then since $\phi_{s}$ is continuous, $P_{\phi}$ a.e., in $s$

$$
\int_{0}^{t} d s h\left(\phi^{(N)}\right)
$$


exists $P_{\phi}$ a.e. as a Riemann integral. Then $\int_{0}^{t} d s h\left(\phi_{s}\right)$ is defined as the convergent limit as $N \rightarrow \infty$ in $L^{p}\left(d P_{\phi}, \Omega\right)$ of the sequence $\int_{0}^{t} d s h\left(\phi^{(N)}\right)$. This follows using (2.29) (2.28) in the region $s>0$ and

$$
E_{\phi}\left(\left|h\left(\phi_{s}\right)\right|^{p}\right) \underset{s \rightarrow 0}{\longrightarrow}|h(\phi)|^{p},
$$

$\mu_{c}$ a.e. in $\phi$. In particular, let $h$ stand for any of the functions $F, G, H$ of (2.25). Then

$$
\begin{gathered}
h\left(\phi_{t}\right) \in L^{p}\left(d P_{\phi}, \Omega\right), \quad 1 \leqq p<\infty, \\
E_{\phi}\left(\int_{0}^{t} d s\left|h\left(\phi_{s}\right)\right|\right)<\infty .
\end{gathered}
$$

We will define the stochastic integral

$$
I_{t}=\int_{0}^{t}\left(: \phi^{3}:, d W_{s}\right)
$$

where $\phi_{s}(W)$ is given by (2.7), as the covergent limit in $L^{2}\left(d P_{\phi}^{(W)}, \Omega\right)$ of the sequence of Ito stochastic integrals

$$
I_{t}^{(N)}=\int_{0}^{t}\left(:\left(\phi_{s}^{(N)}\right)^{3}:, d W^{(N)}\right),
$$

where $W_{s}^{(N)}$ is given by (2.9). Indeed

$$
\begin{aligned}
E_{\phi}^{(W)}\left(\mid I_{t}^{(N+p)}\right. & \left.-\left.I_{t}^{(N)}\right|^{2}\right) \\
& =E_{\phi}\left(\int_{0}^{t} d s\left\|C^{\frac{1-\varepsilon}{2}}:\left(\phi_{s}^{(N+p)}\right)^{3}:-C^{\frac{1-\varepsilon}{2}}:\left(\phi_{s}^{(N)}\right)^{3}:\right\|_{L^{2}(\Lambda)}^{2}\right) \underset{N \rightarrow \infty}{\longrightarrow} 0,
\end{aligned}
$$

since

$$
E_{\phi}\left(\int_{0}^{t} d s\left(: \phi^{3}:, C^{1-\varepsilon}: \phi^{3}:\right)\right)<\infty
$$

by the above.

Now by finite dimensional Ito calculus [13-16],

$$
\begin{aligned}
F\left(\phi_{t}^{(N)}(W)\right) & =F\left(\phi^{(N)}\right)+\int_{0}^{t}\left(:\left(\phi_{s}^{(N)}\right)^{3}:, d W_{s}\right)+\int_{0}^{t} L_{0} F\left(\phi_{s}^{(N)}(W)\right) d s \\
& =F\left(\phi^{(N)}\right)+\int_{0}^{t}\left(:\left(\phi_{s}^{(N)}\right)^{3}:, d W_{s}\right)-\frac{1}{2} \int_{0}^{t} d s G\left(\phi_{s}^{(N)}(W)\right),
\end{aligned}
$$

where we have used (2.25), (2.26). All terms in the above equation converge in $L^{2}\left(d P_{\phi}^{(W)}, \Omega\right)$. Hence taking the limit $N \rightarrow \infty$ in this space,

$$
\frac{1}{4} \int_{\Lambda} d^{2} x: \phi_{t}^{4}:-\frac{1}{4} \int_{A} d^{2} x: \phi^{4}:=\int_{0}^{t}\left(: \phi_{s}^{3}:, d W_{s}\right)-\frac{1}{2} \int_{0}^{t} d s:\left(\phi_{s}^{3}, C^{-\varepsilon} \phi_{s}\right):
$$

Finally let us define the probability measures $Q_{\mu_{c}}^{(W)}, Q_{\mu_{c}}$ on $E \times \Omega$ by

$$
d Q_{\mu_{c}}^{(W)}=d \mu_{c}(\phi) d P_{\phi}^{(W)} \quad \text { and } \quad d Q_{\mu_{c}}=d \mu_{c}(\phi) d P_{\phi}
$$


Here $P_{\phi}^{(W)}, P_{\phi}$ are respectively the Wiener and O.U. measures on $\Omega$. We have of course

$$
\int d Q_{\mu_{c}} h\left(\phi_{t}\right)=\int d Q_{\mu_{c}}^{(W)} h\left(\phi_{t}(W)\right),
$$

where $\phi_{t}(W)$ is given by (2.7).

It is easy to check that if $h \in L^{p}\left(d \mu_{c}\right), 1 \leqq p<\infty$, then

$$
h\left(\phi_{t}(W)\right) \in L^{p}\left(d Q_{\mu_{c}}^{(W)}\right), \quad 1 \leqq p<\infty .
$$

As a consequence $F\left(\phi_{t}\right), G\left(\phi_{t}\right), H\left(\phi_{t}\right)$, where $F, G, H$ are given by (2.25), exist in $L^{p}\left(d Q_{\mu_{c}}^{(W)}\right)$. Moreover the stochastic integral (2.30) as well as the Ito formula (2.31) is valid in $L^{2}\left(d Q_{\mu_{c}}^{(W)}\right)$.

\section{Markov Process Associated}

\section{to the $\left(\phi^{4}\right)_{2}$ Euclidean Field Theory in Finite Volume $\Lambda$}

We consider the stochastic differential equation in $E=H_{-1}(\Lambda)$,

$$
d \hat{\phi}_{t}=d W_{t}-\frac{1}{2}\left(C^{-\varepsilon} \hat{\phi}_{t}+\lambda C^{1-\varepsilon}: \hat{\phi}_{t}^{3}:\right) d t, \quad \hat{\phi}_{0}=\phi,
$$

which can also be written as an integral equation

$$
\hat{\phi}_{t}=\phi_{t}-\frac{\lambda}{2} \int_{0}^{t} d s e^{-\frac{1}{2}(t-s) C^{-\varepsilon}} C^{1-\varepsilon}: \hat{\phi}_{s}^{3}:,
$$

where $\phi_{t}$ is the O.U. process of Sect. 2 .

The vector

$$
\sigma=C^{1-\varepsilon}: \phi^{3}:
$$

belongs to $L^{p}\left(d \mu_{c}, V_{E}\right), 1 \leqq p<\infty$ (see the end of Sect. 1 for the notation), but has no continuity prooerties. Hence the contraction mapping principle (Picard's method) cannot be exploited to solve (3.1) or (3.2).

Instead we will shoot for a weak solution. Namely we will construct a Markov family of measures $\hat{P}_{\phi}, \phi \in E$, on $\Omega=C^{0}([0, \infty), E)$ such that the process

$$
\hat{Z}_{t}=\hat{\phi}_{t}+\frac{\lambda}{2} \int_{0}^{t} d s e^{-\frac{1}{2}(t-s) C^{-\varepsilon}} C^{1-\varepsilon}: \hat{\phi}_{s}^{3}:
$$

(where $\hat{\phi}_{t}$ are paths in $\Omega$ ) has as its $\hat{P}_{\phi}$ joint probability distributions those of the O.U. process $\phi_{t}$ of Sect. 2 . In other words under the law $\hat{P}_{\phi}, \hat{Z}_{t}$ is indistinguishable from $\phi_{t}$, but not necessarily measurable with respect to it.

In this sense $\hat{P}_{\phi}$ solves (3.2). This will be done under the restriction $0<\varepsilon<\frac{1}{10}$.

In this section we will construct the Markov family of measures $\hat{P}_{\phi}$ on $\Omega$, and prove ergodic and mixing properties. Some technical estimates are relegated to the appendix. The unique invariant measure associated to this process is the $\left(\phi^{4}\right)_{2}$ measure of Sect. 1, and in this sense we have "stochastically quantized" Euclidean $\left(\phi^{4}\right)_{2}$ theory in finite volume.

In Sect. 4 we will verify that this family $\hat{P}_{\phi}$ actually gives the claimed weak solution. 
To understand our strategy we first consider the finite dimensional approximation $\phi^{(N)} \rightarrow \phi$, of Sect. 2. Then, in this approximation, with $\phi^{(N)}=\sum_{n=1}^{\mathrm{N}} \phi_{n} e_{n}$ identified also with the vector $\left(\phi_{1}, \ldots, \phi_{N}\right)$ in $R^{N}$ we have

$$
d \hat{\phi}_{t}^{(N)}=d W_{t}^{(N)}-\frac{1}{2}\left(C^{-\varepsilon} \hat{\phi}_{t}^{(N)}+\lambda C^{1-\varepsilon}:\left(\hat{\phi}_{t}^{(N)}\right)^{3}:\right) d t, \quad \hat{\phi}_{0}^{(N)}=\phi^{(N)} .
$$

Note that,

$$
:\left(\phi^{(N)}(x)\right)^{3}:=\left(\phi^{(N)}(x)\right)^{3}-3 C^{(N)}(x) \phi^{(N)}(x)
$$

and $C^{(N)}(x)$ is finite. As a consequence, the drift in (3.4) is a $C^{\infty}$ function on $R^{N}$. Now we can apply the standard method to construct a unique strong solution to (3.4), [13], because we can find an increasing nested sequence of compacts in $R^{N}$ on each of which the drift is bounded and Lipschitz. To make sure that there is no explosion, i.e. the solution is defined for all times, it suffices, [14], to construct a $C^{\infty}$ function $\varrho$ on $R^{N}$, such that it is non-negative and

$$
\varrho_{R}=\inf _{\left\|\phi^{(N)}\right\|>R} \varrho\left(\phi^{(N)}\right) \underset{R \rightarrow \infty}{\longrightarrow} \infty,
$$

where $\left\|\phi^{(N)}\right\|^{2}=\left(\phi^{(N)}, \phi^{(N)}\right)=\sum_{n=1}^{N} \phi_{n}^{2}$,

(ii) there exists a constant $C>0$, such that

$$
L^{(N)} \varrho \leqq C \varrho,
$$

where $L^{(N)}$ is the differential generator associated to (3.4).

In our case it is easy to see that the choice

$$
\varrho\left(\phi^{(N)}\right)=\left\|\phi^{(N)}\right\|_{1-\varepsilon}^{2}+\text { const },
$$

where

$$
\left\|\phi^{(N)}\right\|_{1-\varepsilon}^{2}=\left(\phi^{(N)}, C^{-(1-\varepsilon)} \phi^{(N)}\right)=\sum_{n=1}^{N} \lambda_{n}^{1-\varepsilon} \phi_{n}^{2} \geqq\left\|\phi^{(N)}\right\|^{2}
$$

does the job, using the explicit expression,

$$
\begin{aligned}
L^{(N)=}= & \frac{1}{2} \int_{\Lambda \times \Lambda} d^{2} \times d^{2} y C^{(N) 1-\varepsilon}(x-y) \frac{\delta^{2}}{\delta \phi^{(N)}(x) \delta \phi^{(N)}(y)} \\
& -\frac{1}{2} \int_{\Lambda \times \Lambda} d^{2} x d^{2} y\left(C^{-\varepsilon}(x-y) \phi^{(N)}(y)+\lambda C^{1-\varepsilon}(x-y):\left(\phi^{(N)}\right)^{3}:(y)\right) \frac{\delta}{\delta \phi^{(N)}(x)} .
\end{aligned}
$$

Thus there is a unique, non-explosive solution $\hat{\phi}_{t}^{(N)}$ of (3.4) which is a diffusion process, and the associated semigroup $e^{t L^{(N)}}$

$$
\left(e^{t L^{(N)}} f\right)(\phi)=E_{\phi}^{(W)}\left(f\left(\hat{\phi}_{t}^{(N)}\right)\right)
$$

can be expressed by the Cameron-Martin-Girsanov formula [14] as

$$
\begin{gathered}
\left(e^{t L^{(N)}} f\right)(\phi)=E_{\phi}^{(W)}\left(f\left(\phi_{t}^{(N)}\right) e^{\xi_{t}^{(N)}(W)}\right), \\
\xi_{t}^{(N)}(W)=-\frac{\lambda}{2} \int_{0}^{t}\left(:\left(\phi_{s}^{(N)}\right)^{3}:, d W_{s}^{(N)}\right)-\frac{\lambda^{2}}{8} \int_{0}^{t} d s\left(:\left(\phi_{s}^{(N)}\right)^{3}:, C^{1-\varepsilon}:\left(\phi_{s}^{(N)}\right)^{3}:\right) .
\end{gathered}
$$


In the above $\phi_{t}^{(N)}(W)$ is the unique solution of (3.4), for $\lambda=0$, and is thus the finite dimensional approximation to the O.U. process $\phi_{t}$ of Sect. 2.

We also have,

$$
e^{t L^{(N)}} 1=E_{\phi}^{(W)}\left(e^{\xi(N)}\right)=1,
$$

because of the absence of explosion.

We now proceed to the infinite dimensional system (3.1), (3.2) by simply defining the semigroup $e^{t L}$ by:

$$
\left(e^{t L} f\right)(\phi)=E_{\phi}^{(W)}\left(f\left(\phi_{t}(W)\right) e^{\xi_{t}(W)}\right),
$$

where

$$
\xi_{t}(W)=-\frac{\lambda}{2} \int_{0}^{t}\left(: \phi_{s}^{3}:, d W_{s}\right)-\frac{\lambda^{2}}{8} \int_{0}^{t}\left(: \phi_{s}^{3}:, C^{1-\varepsilon}: \phi_{s}^{3}:\right)
$$

and $\phi_{s}$ is the O.U. process of Sect. 2.

In Sect. 2 we showed that each term in (3.10) belongs to $L^{2}\left(d P_{\phi}^{(W)}, \Omega\right)$. Moreover $\xi_{t}^{(N)} \rightarrow \xi_{t}$ in $L^{2}\left(d P_{\phi}^{(W)}, \Omega\right)$. Thus $\xi_{t}$ is a well defined non-anticipating random variable. Theorem 1, proved below, shows that $e^{t L}$ as defined by (3.9), (3.10) exists for $f$ a bounded measurable function, and moreover $e^{t L} 1=1$. This shows in particular that $e^{t L}$ is a contraction on $L^{\infty}\left(d \mu_{c}\right)$. Theorem 2, proved below, shows that $e^{t L}$ is a strongly continuous, contractive selfadjoint semi-group on $L^{2}(d \mu)$ with 1 as the unique ground state.

First we need

Lemma 1. Let $0<\varepsilon<\frac{1}{10}$. Then,

$$
\begin{gathered}
E_{\phi}^{(W)}\left(e^{p \xi_{t}}\right)<\infty, \quad 1 \leqq p<\infty, \\
E_{\phi}^{(W)}\left(e^{p \xi_{t}^{(N)}}\right)<C_{t, \phi,|A|},
\end{gathered}
$$

where $C_{t, \phi,|\Lambda|}$ is a generic constant depending on $t, \phi,|\Lambda|$ and independent of $N$. Proof.

$$
E_{\phi}^{(W)}\left(e^{p \xi_{t}}\right) \leqq E_{\phi}^{(W)}\left(e^{-p \frac{\lambda}{2} \frac{t}{2}\left(: \phi_{s}^{3}:, d W_{s}\right)}\right) .
$$

Now use the Ito formula (2.31). Hence

$$
\begin{aligned}
& E_{\phi}^{(W)}\left(e^{p \xi_{t}}\right) \leqq e^{\frac{p \lambda}{8} \int_{A}^{2} d^{2} x: \phi_{t}^{4}:} E_{\phi}\left(e^{-\frac{p \lambda}{8} \int_{A} d^{2} x: \phi_{t}^{4}:-\frac{p \lambda t}{4} \int_{0}^{t} d s:\left(\phi^{3}, C^{-\varepsilon} \phi_{s}\right):}\right)
\end{aligned}
$$

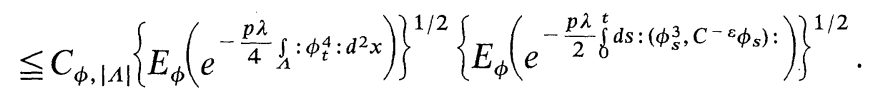

Since $e^{-\frac{p \lambda}{4} \int_{A}: \phi^{4}: d^{2} x}$ belongs to $L^{q}\left(d \mu_{c}\right), 1 \leqq q<\infty$ by Nelson's estimate (Sect. 1 ), it follows by virtue of (2.29) that

$$
E_{\phi}\left(e^{-\frac{p \lambda}{4} \int_{1}: \phi_{t}^{4}: d^{2} x}\right)<\infty
$$


Define

$$
M(\phi)=:\left(\phi^{3}, C^{-\varepsilon} \phi\right): .
$$

It is shown in the appendix that $\exp (-M(\phi)) \in L^{q}\left(d \mu_{c}\right), 1 \leqq q<\infty$, and also that

$$
\exp \left(-\int_{0}^{t} d s M\left(\phi_{\mathrm{s}}\right)\right) \in L^{q}\left(d P_{\phi}, \Omega\right), \quad 1 \leqq q<\infty .
$$

Hence

$$
E_{\phi}^{(W)}\left(e^{p \xi_{t}}\right)<\infty, \quad 1 \leqq p<\infty
$$

which is (i).

Moreover, as stated in the appendix we have (by the method of [31]) not only integrability but also stability bounds for each factor in (3.11): replacing $\phi$ by $\phi^{(N)}$, each of the two factors in (3.11) is uniformly bounded above, independent of $N$. This gives (ii).

Now we proceed to Theorem 1.

Theorem 1. The semi-group $e^{t L}$ is well defined (i.e. the right-hand side of (3.9) exists) for $f$, bounded and measurable. Moreover

$$
e^{t L} 1=1 .
$$

As a consequence, $e^{t L}$ is a contraction on $L^{\infty}\left(d \mu_{c}\right)$.

Proof. That the right-hand side of (3.9) is finite follows from (i) of Lemma 1. Now we prove the next statement

$$
\begin{aligned}
E_{\phi}^{(W)}\left(e^{\xi_{t}}\right) & =E_{\phi}^{(W)}\left(e^{\xi_{t}^{(N)}}\right)+E_{\phi}^{(W)}\left(e^{\xi_{t}}-e^{\xi^{(N)}}\right) \\
& =1+E_{\phi}^{(W)}\left(e^{\xi_{t}}-e_{t}^{\xi_{t}^{(N)}}\right)
\end{aligned}
$$

where we have used (3.8).

Hence

$$
\begin{aligned}
\left|E_{\phi}^{(W)}\left(e^{\xi_{t}}\right)-1\right| & \leqq E_{\phi}^{(W)}\left(\left|e^{\xi_{t}}-e^{\xi_{t}^{(N)}}\right|\right) \leqq E_{\phi}^{(W)}\left(\left|\xi_{t}-\xi_{t}^{(N)}\right|\left|e^{\xi_{t}}+e^{\xi_{t}^{(N)}}\right|\right) \\
& \leqq\left\{E_{\phi}^{(W)}\left(\left|\xi_{t}-\xi_{t}^{(N)}\right|^{2}\right)\right\}^{1 / 2}\left(\left\{E_{\phi}^{(W)}\left(e^{2 \xi_{t}}\right)\right\}^{1 / 2}+\left\{E_{\phi}^{(W)}\left(e^{2 \xi^{(N)}}\right)\right\}^{1 / 2}\right)
\end{aligned}
$$

As $N \rightarrow \infty$, the first factor tends to zero, whereas the second factor is uniformly bounded above by Lemma 1 . Hence, letting $N \rightarrow \infty$

$$
E_{\phi}^{(W)}\left(e^{\xi_{t}}\right)=1
$$

We now turn to Theorem 2.

Theorem 2. Let $e^{t L}$ be defined by (3.9). Then $e^{t L}$ is a bounded self-adjoint, strongly continuous semi-group on $L^{2}(d \mu), d \mu=d \mu_{c} \exp \left(-\frac{\lambda}{4} \int_{A} d^{2} x: \phi^{4}:(x)\right), e^{t L}$ is positivity preserving and improving, and 1 is the unique ground state provided $0<\varepsilon<\frac{1}{10}$.

Remark. Because, $e^{t L}$ is symmetric in $L^{2}(d \mu)$ it follows that

$$
\int d \mu e^{t L} f=\int d \mu f
$$


for $f \in L^{2}(d \mu)$. It follows [12, Chap. XIII] that $e^{t L}$ is not only bounded on $L^{2}(d \mu)$ but also contractive. Hence the infinitesimal generator $L$ is a self-adjoint operator and $-L \geqq 0$.

Proof of the Theorem. Let

$$
U: L^{2}(d \mu) \rightarrow L^{2}\left(d \mu_{c}\right)
$$

be the unitary map given by

$$
f \rightarrow \tilde{f}=U f=e^{-\frac{\lambda}{8} \int_{\Lambda}^{2} d^{2} x: \phi^{4}:(x)} f .
$$

Let $e^{t L}$ be defined by (3.9); then

$$
\begin{aligned}
& \left(f, e^{t L} g\right)_{L^{2}(d \mu)}=\int d \mu_{c}(\phi) e^{-\frac{\lambda}{4} \int_{\Lambda}^{2} d^{2} x: \phi^{4}:(x)} f(\phi) E_{\phi}^{(W)} \\
& \cdot\left(g\left(\phi_{t}(W)\right) e^{\left.-\frac{\lambda}{2} \int_{0}^{t}\left(: \phi_{s}^{3}(W):, d W_{s}\right)-\frac{\lambda^{2} t}{8} \int_{0}^{t} d s \| C^{\frac{1}{2}(1-\varepsilon): \phi_{s}^{3}(W):\|\|^{2}}\right)}\right.
\end{aligned}
$$

We now use the Ito formula (2.31) of Sect. 2. Let $\tilde{f}, \tilde{g}$ be the unitary transform of $f, g$ into $L^{2}\left(d \mu_{c}\right)$ given by (3.12). Then we obtain:

$$
\left(f, e^{t L} g\right)_{L^{2}(d \mu)}=\left(\tilde{f}, e^{t \tilde{L} \tilde{g})_{L^{2}\left(d \mu_{c}\right)}},\right.
$$

where

$$
\begin{gathered}
\left(e^{t \tilde{L}} \tilde{g}\right)(\phi)=E_{\phi}\left(\tilde{g}\left(\phi_{t}\right) e^{-\delta^{t} d s \tilde{V}\left(\phi_{s}\right)}\right), \\
\tilde{V}(\phi)=\frac{\lambda}{4}:\left(\phi^{3}, C^{-\varepsilon} \phi\right):+\frac{\lambda^{2}}{8}\left(: \phi^{3}:, C^{1-\varepsilon}: \phi^{3}:\right) .
\end{gathered}
$$

$\phi_{t}$ is the O.U. process of Sect. 2 and we recall that $0<\varepsilon<\frac{1}{10}$. First note that $\tilde{V}(\phi) \in L^{p}\left(d \mu_{c}\right), 1 \leqq p<\infty$. We show in the appendix that $\exp -\frac{\lambda}{4}:\left(\phi^{3}, C^{-\varepsilon} \phi\right)$ : is in $L^{1}\left(d \mu_{c}\right)$ for any $\lambda>0$.

This fact, together with the fact that the second term in (3.9) is positive, implies that $\exp (-\tilde{V}) \in L^{1}\left(d \mu_{c}\right)$. Moreover the O.U. process $\phi_{t}$ is hyper-contractive (Sect. 2). It follows [2-6], [6] gives the abstract setting used in this paper, that $e^{t \tilde{L}}$ is a strongly continuous bounded self-adjoint semi-group on $L^{2}\left(d \mu_{c}\right)$.

Moreover $\exists T>0$ such that for $t \geqq T, e^{t \tilde{L}}$ is a bounded map from $L^{2}\left(d \mu_{c}\right)$ to $L^{4}\left(d \mu_{c}\right)$, see e.g. [6]. Hence, by virtue of [10], $e^{t \tilde{L}}$ has a ground state with finite multiplicity. It now follows, because of (3.13), that $e^{t L}$ is a strongly continuous bounded self-adjoint semi-group on $L^{2}(d \mu)$. Moreover $e^{t L}$ has a ground state with finite multiplicity.

The representation (3.9) shows that $e^{t L}$ is positivity preserving. We now prove that $e^{t L}$ is positivity improving: i.e. if $f, g, \geqq 0$ are positive vectors in $L^{2}(d \mu)$ then

$$
\left(f, e^{t L} g\right)_{L^{2}(d \mu)}>0
$$

(by a positive vector we mean non-negative and not identically zero). 
If $f, g$ are positive vectors in $L^{2}(d \mu)$, then $\tilde{f}, \tilde{g}$ their unitary transforms via (3.12) are positive vectors in $L^{2}\left(d \mu_{c}\right)$. Then (3.16) will follow, because of (3.13), from

$$
\left(\tilde{f}, e^{t \tilde{L}} \tilde{g}\right)>0 \text {. }
$$

Inequality (3.17) follows from a standard argument [2]. Namely, recall $Q_{\mu_{c}}$ the measure on $E \times \Omega$ given at the end of Sect. 2. Then if $f, g \in L^{2}\left(d \mu_{c}\right)$, are positive vectors, then

$$
\int_{E \times \Omega} d Q_{\mu_{c}} \tilde{f}(\phi) \tilde{g}(\phi)=\left(\tilde{f}, e^{t L_{0}} \tilde{g}\right)_{L^{2}\left(d \mu_{c}\right)}>0
$$

since the O.U. semigroup $e^{t L_{0}}$ is positivity improving. It follows that $\tilde{f}(\phi) \tilde{g}\left(\phi_{t}\right)$ is a positive vector in $\Omega \times E$. Moreover

$$
e^{-\int_{0}^{t} \tilde{V}\left(\phi_{s}\right) d s}>0, \quad Q_{\mu_{c}} \text { a.e. }
$$

Hence

$$
\left(\tilde{f}, e^{t \tilde{L} \tilde{g})_{L^{2}\left(d \mu_{c}\right)}}=\int_{\Omega \times E} d Q_{\mu_{c}} \tilde{f}(\phi) \tilde{g}\left(\phi_{t}\right) e^{-\frac{f}{\delta} \tilde{V}\left(\phi_{s}\right) d s}>0 .\right.
$$

We conclude that $e^{t L}$ is positivity improving. Hence by the Perron-Frobenius argument $[1,2]$ it follows that $e^{t L}$ has a unique ground state. That 1 is the ground state

$$
e^{t L} 1=1
$$

follows from Theorem 1.

Remark. Theorem 2 implies by standard results [12] that the semi-group $e^{t L}$ is ergodic. In other words, if $f \in L^{2}(d \mu)$, and $Z=\int d \mu$,

$$
\lim _{T \rightarrow \infty} \frac{1}{T} \int_{0}^{T} d s\left(e^{s L} f\right)(\phi)=\frac{1}{Z} \int d \mu(\phi) f(\phi), \quad \mu \text { a.e. }
$$

However, in our case we have a stronger result, namely that $e^{t L}$ is mixing:

$$
\lim _{t \rightarrow \infty}\left(f, e^{t L} g\right)_{L^{2}(d \mu)}=\frac{1}{Z}\left(\int d \mu f\right)\left(\int d \mu g\right) .
$$

This follows from the fact that $e^{t L}$ has a mass gap.

The existence of the mass gap follows from the following facts (for details see $[4,6])$ : a) the resolvent of the O.U. generator $R_{0}=\left(\lambda-L_{0}\right)^{-1}$ in a finite box is compact; b) the resolvent $\widetilde{R}=(\lambda-\widetilde{L})^{-1}$ is also compact as $\widetilde{V}$ is an almost semibounded perturbation of $L_{0}$ and $L_{0}$ generates a hypercontractive semigroup; c) $e^{t \tilde{L}}$ has a ground state. Hence $e^{t L}$ has a mass gap.

The Markov Process $\hat{\phi}_{t}$

The previous Theorems 1 and 2 and the representation (3.5) enables us to construct the desired ergodic Markov process $\hat{\phi}_{t}$ which gives the stochastic quantization of continuum $\left(\phi^{4}\right)_{2}$ Euclidean field theory in finite volume. 
We define transition probabilities $\hat{p}_{t}\left(\phi, d \phi^{\prime}\right)$ as probability measures on $E=H_{-1}(\Lambda)$ by:

$$
\hat{p}_{t}(\phi, B)=E_{\phi}^{(W)}\left(\chi\left\{\phi_{t}(W) \in B\right\} e^{\left.-\frac{\lambda}{2} \delta^{t}\left(: \phi_{s}^{3}(W):, d W_{s}\right)-\frac{\lambda^{2}}{8} \int_{0}^{t} d s \| C^{\frac{1}{2}(1-\varepsilon): \phi_{s}^{3}(W): \|^{2}}\right),}\right.
$$

where $\chi$ is the characteristic function of an event and $B$ is a Borel set in $E$.

The exponential in (3.21) is integrable by virtue of Theorem 1. Moreover $e^{t L} 1=1$. From the countable additivity of $E_{\phi}^{(W)}$ it now follows that $\hat{p}_{t}(\phi, \cdot)$ is a family of countably additive probability measures on $E$. Moreover the theorems assure us that $\hat{p}_{t}(\phi, B)$ is $\mu_{c}$ measurable in $\phi$, and $d t$ measurable in $t$.

Hence $\hat{p}_{t}(\phi, B)$ are "stochastic kernels" and qualify as transition probabilities of a Markov process $\hat{\phi}_{t}$ with values in $E$. Its joint probability distributions are defined in the standard way: $\left(0<t_{1}<\ldots<t_{n}\right)$

$$
\begin{aligned}
& \hat{p}_{\phi}\left\{\hat{\phi}_{t_{1}} \in B_{1}, \ldots, \hat{\phi}_{t_{n}} \in B_{n}\right\} \\
& \quad=\int_{B_{1}} \ldots \int_{B_{n}} \hat{p}_{t_{1}}\left(\phi, d \phi_{1}\right) \hat{p}_{t_{2}-t_{1}}\left(\phi_{1}, d \phi_{2}\right) \ldots \hat{p}_{t_{n}-t_{n-1}}\left(\phi_{n-1}, d \phi_{n}\right) \\
& \quad=\hat{p}_{t_{1} \ldots t_{n}}\left(B_{1} \times B_{2} \times \ldots \times B_{n}\right),
\end{aligned}
$$

which defines $\hat{p}_{t_{1} \ldots t_{n}}$ as a consistent family of probability measures on $(E)^{n}$. We shall show later that $\hat{P}_{\phi}$ can be realized as probability measures on $\Omega$, our path space, and then $\left(\Omega, \hat{p}_{\phi}\right)$ constitutes our Markov process with continuous sample paths. Let us note, from (3.22), that if $f_{1}, \ldots, f_{n}$ are test functions,

$$
\begin{aligned}
\hat{E}_{\phi}\left(\hat{\phi}_{t_{1}}\left(f_{1}\right) \ldots \hat{\phi}_{t_{n}}\left(f_{n}\right)\right)= & E_{\phi}^{(W)}\left(\phi _ { t _ { 1 } } ( f _ { 1 } ) \ldots \phi _ { t _ { n } } ( f _ { n } ) \operatorname { e x p } \left\{-\frac{\lambda}{2} \int_{0}^{t_{n}}\left(: \phi_{s}^{3}(W):, d W_{s}\right)\right.\right. \\
& \left.\left.-\frac{\lambda^{2}}{8} \int_{0}^{t_{n}} d s\left\|C^{\frac{1}{2}(1-\varepsilon)}: \phi_{s}^{3}(W)\right\|^{2}\right\}\right) .
\end{aligned}
$$

By the preceding remark after Theorem $2, e^{t L}$ is mixing. Hence we have:

$$
\lim _{t \rightarrow \infty} \hat{E}_{\phi}\left(\hat{\phi}_{t_{1}}\left(f_{1}\right) \ldots \hat{\phi}_{t_{n}}\left(f_{n}\right)\right)=\frac{1}{Z} \int d \mu(\phi) \phi\left(f_{1}\right) \ldots \phi\left(f_{n}\right), \quad \mu \text { a.e. }
$$

which is the aim of stochastic quantization of the $\left(\phi^{4}\right)_{2}$ theory.

We shall now show that the Markov process $\hat{\phi}_{t}$ constructed from its transition probabilities has a continuous version. In other words, there exists $\mu$ a.e. in $\phi$ a Markovian family of probability measures $\hat{P}_{\phi}$ on $\Omega=C^{0}\left([0, \infty), E=H_{-1}(\Lambda)\right)$ of which (3.22) are the joint probability distributions. For convenience, we run off the Markov process $\hat{\phi}_{t}$ with initial distribution $\mu$. We shall prove:

Proposition. Let $0<s<t<T$. Then

$$
\int_{E} d \mu(\phi) \hat{E}_{\phi}\left(\left\|\hat{\phi}_{t}-\hat{\phi}_{s}\right\|_{E}^{4}\right) \leqq C|t-s|^{2}\left(C_{T}\right)^{t-s} .
$$

Corollary. By virtue of the estimate (3.25), Kolmogoroff's theorem, see e.g. [14], assures us that there exists a probability measure $\hat{Q}_{\mu}$ on $\Omega=C^{0}([0, \infty), E)$ such that, if $B_{1}, \ldots, B_{n}$ are Borel sets in $E$,

$$
\begin{aligned}
& \hat{Q}_{\mu}\left\{\hat{\phi}_{t_{1}} \in B_{1}, \ldots, \hat{\phi}_{t_{n}} \in B_{n}\right\} \\
& \quad=\int_{E} d \mu(\phi) \int_{B_{1}} \ldots \int_{B_{n}} \hat{p}_{t_{1}}\left(\phi, d \phi_{1}\right) \hat{p}_{t_{2}-t_{1}}\left(\phi_{1}, d \phi_{2}\right) \ldots \hat{p}_{t_{n}-t_{n-1}}\left(\phi_{n-1}, d \phi_{n}\right),
\end{aligned}
$$


$\left(\hat{Q}_{\mu}, \Omega\right)$ is a stationary Markov process. We can write

$$
\hat{Q}_{\mu}(B)=\int d \mu(\phi) \hat{p}_{\phi}(B),
$$

where $B$ is a Borel set in $\Omega$. $\left(\hat{P}_{\phi}, \Omega\right)$ is the Markov process started off at $\phi$.

Proof of the Proposition. It is straightforward to verify that the continuous O.U. process $\phi_{t}$ of Sect. 2 satisfies

$$
\int \mathrm{d} \mu_{c}(\phi) E_{\phi}\left(\left\|\phi_{r}-\phi_{s}\right\|_{E}^{2 r}\right) \leqq C|t-s|^{r}
$$

for any positive integer $r \geqq 1$. Next we note

$$
\begin{aligned}
\int d \mu(\phi) \hat{E}_{\phi}\left(\left\|\hat{\phi}_{t}-\hat{\phi}_{s}\right\|^{2 r}\right) & =\int d \mu(\phi) \int \hat{p}_{s}\left(\phi, d \phi_{1}\right) \int \hat{p}_{t-s}\left(\phi_{1}, d \phi_{2}\right)\left\|\phi_{1}-\phi_{2}\right\|_{E}^{2 r} \\
& =\int d \mu(\phi) \int \hat{p}_{t-s}\left(\phi, d \phi_{2}\right)\left\|\phi-\phi_{2}\right\|_{E}^{2 r},
\end{aligned}
$$

where we have used the fact that $e^{t L}$ leaves $\mu$ invariant. Hence $\left(\phi_{t}\right.$ is the O.U. process)

$$
\begin{aligned}
& \int d \mu(\phi) E_{\phi}\left(\left\|\hat{\phi}_{t}-\hat{\phi}_{s}\right\|_{E}^{2 r}\right)=\int d \mu(\phi) E_{\phi}^{(W)}\left(\left\|\phi-\phi_{t-s}(W)\right\|_{E}^{2 r}\right. \\
& \left.\cdot \exp \left\{-\frac{\lambda}{2} \int_{0}^{t-s}\left(: \phi_{s_{1}}^{3}(W):, d W_{s_{1}}\right)-\frac{\lambda^{2}}{8} \int_{0}^{t-s} d s_{1}\left\|C^{\frac{1}{2}(1-\varepsilon)}: \phi_{s_{1}}^{3}(W):\right\|^{2}\right\}\right) \\
& =\int d \mu_{c}(\phi) E_{\phi}^{(W)}\left(\| \phi - \phi _ { t - s } ( W ) \| _ { E } ^ { 2 r } \operatorname { e x p } \left\{-\frac{\lambda}{4} \int_{A} d^{2} x: \phi^{4}:(x)\right.\right. \\
& \left.\left.\quad-\frac{\lambda}{2} \int_{0}^{t-s}\left(: \phi_{s_{1}}^{3}:, d W_{s_{1}}\right)-\frac{\lambda^{2}}{8} \int_{0}^{t-s} d s_{1}\left\|C^{\frac{1}{2}(1-\varepsilon)}: \phi_{s_{1}}^{3}:\right\|^{2}\right\}\right)
\end{aligned}
$$

and, on using the Ito formula (2.31)

$$
\begin{aligned}
= & \int d \mu_{c}(\phi) E_{\phi}\left(\| \phi - \phi _ { r - s } \| _ { E } ^ { 2 r } \operatorname { e x p } \left\{-\frac{\lambda}{8} \int_{\Lambda} d^{2} x: \phi^{4}:(x)-\frac{\lambda}{8} \int_{\Lambda} d^{2} x: \phi_{t-s}^{4}:(x)\right.\right. \\
& \left.\left.-\int_{0}^{t-s} d s_{1} \tilde{V}\left(\phi_{s_{1}}\right)\right\}\right),
\end{aligned}
$$

where $\tilde{V}(\phi)$ is given by (3.15). Apply Hölder's inequality

$$
\begin{aligned}
& \leqq\left(\int d \mu_{c}(\phi) E_{\phi}\left(\left\|\phi-\phi_{t-s}\right\|_{E}^{8 r}\right)\right)^{1 / 4} \cdot\left(\int d \mu_{c}(\phi) E_{\phi}\left(e^{-\frac{\lambda}{2} \int_{\Lambda}: \phi^{4}:(x) d^{2} x}\right)\right)^{1 / 4} \\
& \cdot\left(\int d \mu_{c}(\phi) E_{\phi}\left(e^{-\frac{\lambda}{2} \int_{A}: \phi_{t-s}:(x) d^{2} x}\right)\right)^{1 / 4} \cdot\left(\int d \mu_{c}(\phi) E_{\phi}\left(e^{-4 \int_{0}^{t-s} \tilde{V}\left(\phi_{s_{1}}\right) d s_{1}}\right)\right)^{1 / 4} \\
& \leqq\left(\int d \mu_{c}(\phi) E_{\phi}\left(\left\|\phi-\phi_{t-s}\right\|_{E}^{8 r}\right)\right)^{1 / 4} \\
& \cdot\left\|e^{-\frac{\lambda}{4} \int_{\Lambda}^{d^{2} x: \phi^{4}:(x)}}\right\|_{L^{2}\left(d \mu_{c}\right)} \cdot\left\|e^{-4(t-s) \tilde{V}}\right\|_{L^{1}\left(d \mu_{c}\right)}^{14},
\end{aligned}
$$

where the last factor in (3.28) has been estimated using the Riemann sum approximation for $\int_{0}^{t-s} d s_{1} \ldots$, Hölder's inequality (see e.g. [5]) and that $e^{t L_{0}}$ leaves 
$\mu_{c}$ invariant. The latter was also used for the second factor. It is easy to check

$$
\int d \mu_{c}(\phi) E_{\phi}\left(\left\|\phi_{t}-\phi_{s}\right\|_{E}^{8 r}\right)=\int d \mu_{c}(\phi) E_{\phi}\left(\left\|\phi-\phi_{t-s}\right\|_{E}^{8 r}\right),
$$

and

$$
\left\|e^{-4(r-s) \tilde{V}}\right\|_{L^{\prime}\left(d \mu_{c}\right)} \leqq\left(\left\|e^{-\tilde{V}}\right\|_{L^{4 T}\left(d \mu_{c}\right)}\right)^{t-s} .
$$

From (3.29), (3.31), and (3.27),

$$
\int d \mu(\phi) \hat{E}_{\phi}\left(\left\|\hat{\phi}_{t}-\hat{\phi}_{s}\right\|^{2 r}\right) \leqq C|t-s|^{r}\left(C_{T}\right)^{t-s},
$$

choosing $r=2$ in (3.32), the proof of the proposition is complete.

\section{The Process $\hat{\phi}_{t}$ as a Weak Solution of (3.2)}

It is legitimate to ask in what sense the ergodic Markov process $\left(\Omega, \hat{P}_{\phi}\right)$ constructed in Sect. 3 solves Eq. (3.2), which was our starting point.

Let $\Omega_{T}=C^{0}([0, T], E)$ and $\hat{P}_{\phi}$ the Markov family of measures on $\Omega_{T}$ obtained from the joint probability distributions (3.22) by what we have shown previously. Then the answer to the question is given by the following proposition.

\section{Proposition.}

$$
\hat{Z}_{t}=\hat{\phi}_{t}+\frac{\lambda}{2} \int_{0}^{t} d s e^{-\frac{(t-s)}{2} c^{-\varepsilon}} C^{1-\varepsilon}: \hat{\phi}_{s}^{3}:
$$

has the same joint probability distributions (with respect to $\left(\Omega_{T}, \hat{P}_{\phi}\right)$ ) as the O.U. process $\phi_{t}$ of Sect. 2. Thus we have a weak solution of (3.2). Note that $\hat{Z}_{t}$ which can be identified as an O.U. process is not necessarily measurable with respect to $\phi_{t}$.

Proof. We merely have to show that the transition probabilities of $\hat{Z}_{t}$, with respect to $\hat{P}_{\phi}$, are that of the O.U. process $\phi_{t}$. Let $\hat{E}_{\phi}$ be the expectation with respect to $\hat{P}_{\phi}$ and $f$ a bounded measurable function on $E$. We have from (3.5)

$$
\hat{E}_{\phi}\left(f\left(\hat{Z}_{t}\right)\right)=E_{\phi}^{(W)}\left(f\left(Z_{t}\right) e^{\xi_{0}^{t}}\right)
$$

where

$$
\begin{gathered}
Z_{t}=\phi_{t}+\frac{\lambda}{2} \int_{0}^{t} d s e^{-\frac{(t-s)}{2} C^{-\varepsilon}} C^{1-\varepsilon}: \phi_{s}^{3}:, \\
\xi_{s}^{t}=-\frac{\lambda}{2} \int_{s}^{t}\left(: \phi_{s}^{3}:, d W_{s}\right)-\frac{\lambda^{2}}{8} \int_{s}^{t} d s\left\|C^{1 / 2(1-\varepsilon)}: \phi_{s}^{3}(W):\right\|^{2},
\end{gathered}
$$

and $\phi_{t}(W)$ is the O.U. process, starting at $\phi$, given by (2.7). By virtue of Theorem 1 of Sect. 3

$$
e^{\xi_{0}^{T}} \in L^{1}\left(\Omega_{T}, d P^{(W)}\right)
$$

and moreover

$$
E_{\phi}^{(W)}\left(e^{\xi_{0}^{T}}\right)=1
$$


Let $\mathbb{B}_{t}$ be the $\sigma$-subalgebra engendered by $W_{s}, \forall s \leqq t$. Then it is a straightforward consequence of (4.6) (see e.g. Lemma 2.3, Chap. 7, [16]) that the conditional expectation

$$
E_{\phi}\left(e^{\xi_{s}^{t}} \mid \mathbb{B}_{s}\right)=1, \quad P^{(W)} \text { a.s. }
$$

Define on $\Omega_{T}$ the probability measure $\widetilde{P}^{(W)}$ by

$$
d \widetilde{P}_{\phi}^{(W)}=e^{\xi_{0}^{T}} d P_{\phi}^{(W)},
$$

and let $\widetilde{E}_{\phi}^{(W)}$ be expectation with respect to $\tilde{P}_{\phi}^{(W)}$.

Note that, using (4.8) and (4.7),

$$
\begin{aligned}
\tilde{E}_{\phi}^{(W)}\left(f\left(Z_{t}\right)\right. & =E_{\phi}^{(W)}\left(f\left(Z_{t}\right) e^{\xi_{0}^{T}}\right)=E_{\phi}^{(W)}\left(f\left(Z_{t}\right) e^{\xi_{\xi^{t}}^{t}} e^{\xi_{t}^{T}}\right) \\
& =E_{\phi}^{(W)}\left(f\left(Z_{t}\right) e^{\xi_{5}^{t}} E_{\phi}\left(e^{\xi_{t}} \mid \mathbb{B}_{t}\right)\right)=E_{\phi}^{(W)}\left(f\left(Z_{t}\right) e^{\xi_{0}^{t}}\right) .
\end{aligned}
$$

Hence from (4.2) and (4.9) we have,

$$
\hat{E}_{\phi}\left(f\left(\hat{Z}_{t}\right)=\widetilde{E}_{\phi}^{(W)}\left(f\left(Z_{t}\right)\right) .\right.
$$

Thus to prove our proposition we have to show that $Z_{t}$ given by (4.3), is an O.U. process, with respect to the measure $\tilde{P}_{\phi}^{(W)}$, whose joint probability distributions coincide with that of the O.U. process $\phi_{t}^{(W)}$ of Sect. 2.

Because of (4.5) and (4.6) we are assured by the Girsanov theorem [valid in our context because of (4.6)], that ( $W_{t}$ is the Wiener process of Sect. 2)

$$
\tilde{W}_{t}=W_{t}+\frac{\lambda}{2} \int_{0}^{t} d s C^{1-\varepsilon}: \phi_{s}^{3}(W):
$$

is also a Wiener process in $\Omega_{T}$ with respect to the measure $\tilde{P}^{(W)}$ with the same covariance as $W_{t}$

$$
\tilde{E}^{(W)}\left(\tilde{W}_{t}(f) \tilde{W}_{s}(g)\right)=\left(f, C^{1-\varepsilon} g\right) \min (t, s) .
$$

Then, $Z_{t}$ is just the unique solution of

$$
d Z_{t}=d \tilde{W}_{t}-\frac{1}{2} C^{-\varepsilon} Z_{t} d t, \quad Z_{0}=\phi .
$$

Indeed, the solution of (4.13) is:

$$
\begin{aligned}
Z_{t} & =e^{-\frac{t}{2} C^{-\varepsilon}} \phi+\int_{0}^{t} d s e^{-\frac{(t-s)}{2} C^{-\varepsilon}} d \tilde{W}_{s} \\
& =e^{-\frac{t}{2} C^{-\varepsilon}} \phi+\int_{0}^{t} d s e^{-\frac{1}{2}(t-s) C^{-\varepsilon}} d W_{s}+\frac{\lambda}{2} \int_{0}^{t} d s e^{-\frac{1}{2}(r-s) C^{-\varepsilon}} C^{1-\varepsilon}: \phi_{s}^{3}(W): \\
& =\phi_{t}(W)+\frac{\lambda}{2} \int_{0}^{t} d s e^{-\frac{1}{2}(t-s) C^{-\varepsilon}} C^{1-\varepsilon}: \phi_{s}^{3}(W):,
\end{aligned}
$$

which is just (4.3). Comparing (4.13) and the differential equation corresponding to (2.7) we see that $Z_{t}$ is with respect to $\tilde{P}^{(W)}$ an O.U. process with the same joint probability distributions as $\phi_{t}$ of Sect. 2. This proves the proposition. 


\section{Appendix}

Define

$$
M \equiv:\left(\phi^{3}, C^{-\varepsilon} \phi\right):
$$

In this appendix, $C$ will be the covariance with free boundary conditions. In fact by Theorem VII.9 in [2], Propositions 1 and 2 below imply similar estimates for our field with Dirichlet boundary conditions in $\Lambda$.

Then in this appendix we will prove the

Proposition 1. For any $\lambda>0$ and for $\varepsilon$ restricted to the range $0<\varepsilon<\frac{1}{10}$,

$$
e^{-\lambda M} \in L^{1}\left(d \mu_{c}\right) \text {. }
$$

This result was used in the proof of Theorems 1 and 2 in Sect. 3.

Note that $M \in L^{p}\left(d \mu_{c}\right) 1 \leqq p<\infty$, for $\varepsilon<\frac{1}{2}$. The restriction $\varepsilon>0$ was imposed in Sect. 2, to ensure that the transition probabilities of the O.U. process are absolutely continuous with respect to $\mu_{c}$. The upper bound $\varepsilon<\frac{1}{10}$ turns out to be sufficient for (A.2) to hold.

The proof of the above proposition and (A.2) is based on a series of lemmata.

Lemma 1. For $0<\varepsilon<1$,

$$
\left(\phi^{3}, C^{-\varepsilon} \phi\right) \geqq \int_{\Lambda} d^{2} x \phi^{4}(x) .
$$

Proof. We let $\|\cdot\|_{p}$ denote the $L^{p}(\Lambda)$ norm.

Define

$$
a_{\varepsilon}=\int_{0}^{\infty} d s \frac{s^{-1+2 \varepsilon}}{1+s^{2}}>0
$$

which converges for $0<\varepsilon<1$.

We have the representation, converging for $0<\varepsilon<1$,

$$
(-\Delta+1)^{\varepsilon}=a_{\varepsilon}^{-1} \int_{0}^{\infty} d s s^{-1+2 \varepsilon}\left(I-s^{2}\left(-\Delta+1+s^{2}\right)^{-1}\right) .
$$

Hence

$$
\begin{aligned}
\left(\phi^{3},(-\Delta+1)^{\varepsilon} \phi\right) & =a_{\varepsilon}^{-1} \int_{0}^{\infty} d s s^{-1+2 \varepsilon}\left(\|\phi\|_{4}^{4}-s^{2}\left(\phi^{3}, C_{s^{2}+1} \phi\right)\right) \\
& \geqq \mathrm{a}_{\varepsilon}^{-1} \int_{0}^{\infty} d s s^{-1+2 \varepsilon}\left(\|\phi\|_{4}^{4}-s^{2}\left|\left(\phi^{3}, C_{s^{2}+1} \phi\right)\right|\right),
\end{aligned}
$$

where

$$
C_{s^{2}+1}=\left(-\Delta+1+s^{2}\right)^{-1} .
$$

Using Hölder's inequality,

$$
\left|\left(\phi^{3}, C_{s^{2}+1} \phi\right)\right| \leqq\|\phi\|_{4}^{3}\left\|C_{s^{2}+1} \phi\right\|_{4} .
$$


Now use Young's convolution inequality

$$
\|f * \phi\|_{p} \leqq\|f\|_{q}\|\phi\|_{r}, \quad \frac{1}{p}=\frac{1}{q}+\frac{1}{r}-1,
$$

with the choice $f=$ integral kernel of $C_{s^{2}+1}, p=r=4, q=1$.

Note that

$$
\|f\|_{1} \leqq \frac{1}{s^{2}+1} .
$$

Hence

$$
\left\|C_{s^{2}+1} \phi\right\|_{4} \leqq \frac{1}{s^{2}+1}\|\phi\|_{4} .
$$

From (A.5), (A.6), we have

$$
\left|\left(\phi^{3}, C_{s^{2}+1} \phi\right)\right| \leqq \frac{1}{s^{2}+1}\|\phi\|_{4}^{4} .
$$

From (A.4), (A.7)

$$
\left(\phi^{3},(-\Delta+1)^{\varepsilon} \phi\right) \geqq a_{\varepsilon}^{-1} \int_{0}^{\infty} d s s^{-1+2 \varepsilon}\left(1-\frac{s^{2}}{s^{2}+1}\right)\|\phi\|_{4}^{4}=\|\phi\|_{4}^{4} .
$$

Next we turn to Lemma 2.

Define UV cutoff fields $\phi_{\kappa}(x)$ by

$$
\phi_{\kappa}(x)=\int_{|K| \leqq \kappa} \frac{d^{2} K}{(2 \kappa)^{2}} e^{i K x} \tilde{\varphi}(K) .
$$

Define

$$
M_{\kappa}=:\left(\phi_{\kappa}^{3}, C^{-\varepsilon} \phi_{\kappa}\right):=:\left(\phi_{\kappa}^{3},(-\Delta+1)^{\varepsilon} \phi_{\kappa}\right): .
$$

Then for $0<\varepsilon<\frac{1}{2}$

$$
M_{\kappa} \underset{\kappa \rightarrow \infty}{\longrightarrow} M, \text { in } L^{p}\left(d \mu_{c}\right), \quad 1 \leqq p<\infty .
$$

Undoing the Wick ordering,

$$
\begin{aligned}
M_{\kappa}= & \left(\phi_{\kappa}^{3},(-\Delta+1)^{\varepsilon} \phi_{\kappa}\right)-3\left(C_{\kappa}(0)\left(\phi_{\kappa},(-\Delta+1)^{\varepsilon} \phi_{\kappa}\right)\right. \\
& \left.+C_{\kappa}^{1-\varepsilon}(0)\left(\phi_{\kappa}, \phi_{\kappa}\right)\right)+3|\Lambda| C_{\kappa}(0) C_{\kappa}^{1-\varepsilon}(0),
\end{aligned}
$$

where $C_{\kappa}(0)=C_{\kappa}(0,0)$ and $C_{\kappa}(x, y)$ is the integral kernel of $C_{\kappa}$ the covariance of $\phi_{\kappa}, C_{\kappa}^{1-\varepsilon}(0)=C_{\kappa}^{1-\varepsilon}(0,0)$, and $C_{\kappa}^{1-\varepsilon}(0,0)$ is the integral kernel of $C_{\kappa}^{1-\varepsilon}$.

Now use primitive positivity, i.e. Lemma 1 , and

$$
\left(\phi_{\kappa},(-\Delta+1)^{\varepsilon} \phi_{\kappa}\right) \leqq\left(\kappa^{2}+1\right)^{\varepsilon}\left(\phi_{\kappa}, \phi_{\kappa}\right)
$$

to obtain from (A.9)

$$
\begin{aligned}
M_{\kappa} \geqq \int_{A} d^{2} x\left(\left(\phi_{\kappa}(x)\right)^{4}-3\left(C_{\kappa}(0)\left(\kappa^{2}+1\right)^{\varepsilon}+C_{\kappa}^{1-\varepsilon}(0)\right)\left(\phi_{\kappa}(x)\right)^{2}\right. \\
\left.\quad+3 C_{\kappa}(0) C_{\kappa}^{1-\varepsilon}(0)\right) .
\end{aligned}
$$


Now use

$$
C_{\kappa}(0) \sim O(\ln \kappa), \quad C_{\kappa}^{1-\varepsilon}(0) \sim O\left(\kappa^{2 \varepsilon} \ln \kappa\right)
$$

to obtain immediately:

\section{Lemma 2.}

$$
M_{\kappa} \geqq-\operatorname{const} O\left(\kappa^{4 \varepsilon}(\ln \kappa)^{2}\right) .
$$

Define

$$
\tilde{M}_{\kappa} \equiv M-M_{\kappa} .
$$

Then it is straightforward to verify via Feynman graph calculations

\section{Lemma 3.}

$$
\int d \mu_{c}\left|M_{\kappa}\right|^{2 j} \leqq(j !)^{4} b^{j}\left((\ln \kappa)^{m} \kappa^{-2+4 \varepsilon}\right)^{j}
$$

for any $j$, some $m>0 . b$ is a constant independent of $j$ and $\kappa$.

The proof of the proposition at the beginning of the appendix now follows from Lemmata 2 and 3 by Nelson's argument [1-3].

Namely, we have

$$
\begin{aligned}
\mu_{c}\left\{M \leqq-\operatorname{const}\left(\kappa^{4 \varepsilon}(\ln \kappa)^{2}-1\right\}\right. & \leqq \mu_{c}\left\{\left|\tilde{M}_{\kappa}\right|^{2 j} \geqq 1\right\} \leqq \int d \mu_{c}\left|\tilde{M}_{\kappa}\right|^{2 j} \\
& \leqq(j !)^{4} b^{j}\left((\ln \kappa)^{m} \kappa^{-2+4 \varepsilon}\right)^{j}
\end{aligned}
$$

Using Stirling's approximation for $j$ ! and an optimal $\kappa$-dependent choice of $j$, we have

$$
\mu_{c}\left\{M \leqq-\operatorname{const}\left(\kappa^{4 \varepsilon}(\ln \kappa)^{2}\right)-1\right\} \leqq e^{-\operatorname{const} \kappa \frac{2-4 \varepsilon}{4}(\ln \kappa)^{-m / 4}} .
$$

This estimate, together with Lemma 2, assures us that for $\varepsilon<\frac{1}{10} e^{-\lambda M} \in L^{1}\left(d \mu_{c}\right)$, $\lambda>0$, and the proposition has been proved.

Remark. It can also be shown by methods very similar to [31], that we have a uniform bound

$$
\int d \mu_{\mathrm{c}} e^{-\lambda M_{\kappa}} \leqq e^{\mathrm{const}|\Lambda|} .
$$

In Sect. 2, we verified that

$$
\int_{0}^{t} d s M\left(\phi_{s}\right)
$$

with $M$ defined by (A.1) is a random variable in $L^{p}\left(d P_{\phi}, \Omega\right), 1 \leqq p<\infty$ and $P_{\phi}$ is the O.U. measure.

Now $p_{t}\left(\phi, d \phi^{\prime}\right)$, the transition probability of the O.U. process, is absolutely continuous with respect to $\mu_{c}$, for every $t>0$ and starting point $\phi$, and its RadonNikodym derivative is in $L^{2}\left(d \mu_{c}\right)$. This fact, and the proof of Proposition 1 of this appendix leads to, 
Proposition 2. For any $\lambda>0$, and provided $0<\varepsilon<\frac{1}{10}$,

$$
E_{\phi}\left(e^{-\lambda \int_{0}^{t} d s M\left(\phi_{s}\right)}\right)<\infty
$$

where $E_{\phi}$ is expectation with respect to the O.U. measure $P_{\phi}$.

Proof. Define

$$
M^{t}=\int_{0}^{t} d s M\left(\phi_{s}\right), \quad M_{\kappa}^{t}=\int_{0}^{t} d s M_{\kappa}\left(\phi_{s}\right),
$$

$M_{\kappa}$ as in (A.8). Then from Lemma 2,

$$
M_{\kappa}^{(t)} \geqq-\operatorname{const} t O\left(\kappa^{4 \varepsilon}(\ln \kappa)^{2}\right) .
$$

Analogous to the step before Lemma 3, define

$$
\tilde{M}_{\kappa}^{(t)}=M^{(t)}-M_{\kappa}^{(t)}=\int_{0}^{t} d s \tilde{M}_{\kappa}\left(\phi_{s}\right)
$$

Lemma 4.

$$
E_{\phi}\left(\left|\tilde{M}_{\kappa}^{(t)}\right|\right)^{2 j} \leqq C_{\phi}^{t}((2 j) !)^{2} b_{t}^{j}\left((\ln \kappa)^{m} \kappa^{-2+4 \varepsilon}\right)^{j}
$$

for any $j$, some $m>0$, and large $\kappa$.

Note that for large $j$, by Stirling approximation, $((2 j) !)^{2} \sim(2 j)^{4 j}$ whereas $(j !)^{4} \sim j^{4 j}$ so that (for large $j$ ) Lemma 3 and Lemma 4 are the same, as are Lemma 2 and (A.17), $(t$, is fixed).

This Suffices to Prove Proposition 2. It remains to prove Lemma 4.

We have

$$
\begin{aligned}
E_{\phi}\left(\left|\tilde{M}_{\kappa}^{(t)}\right|^{2 j}\right) & \leqq t^{2 j-1} \int_{0}^{t} d s E_{\phi}\left(\left|\tilde{M}_{\kappa}\left(\phi_{s}\right)\right|^{2 j}\right) \\
& \leqq t^{2 j-1} \int_{0}^{t} d s \int p_{s}\left(\phi, d \phi^{\prime}\right)\left|\tilde{M}_{\kappa}\left(\phi^{\prime}\right)\right|^{2 j} \\
& \leqq t^{2 j-1} C_{\phi}^{t}\left(\int_{0}^{t} d s \int p_{s}\left(0, d \phi^{\prime}\right)\left|\tilde{M}_{\kappa}\left(\phi^{\prime}\right)\right|^{4 j}\right)^{1 / 2}
\end{aligned}
$$

where we have used the fact that $p_{s}\left(\phi, d \phi^{\prime}\right)$ is absolutely continuous with respect to $p_{s}\left(0, d \phi^{\prime}\right)$ and the radon-Nikodym derivative is in $L^{2}\left(p_{s}\left(0, d \phi^{\prime}\right)\right)$, and Schwarz inequality.

Now $p_{t}\left(0, d \phi^{\prime}\right)$ is a gaussian measure with mean 0 and covariance

$$
C_{t}=\left(1-e^{-t C^{-\varepsilon}}\right) C,
$$

and we have for integral kernels

$$
\lim _{x \rightarrow y}\left(C_{t}(x, y)-C(x, y)\right)=\delta C_{t}<\infty .
$$

$\tilde{M}_{\kappa}(\phi)$ has Wick ordering with respect to $C$. By virtue of (A.22) we can change the Wick ordering in $\tilde{M}_{\kappa}(\phi)$ to $C_{s}$-Wick ordering at the cost of introducing lower order terms with finite coefficients [1]. 
Now, because of (A.21), we have by Feynman graph calculations exactly as leading to Lemma 3, for large $\kappa$

$$
\int_{0}^{t} d s \int p_{s}\left(0, d \phi^{\prime}\right)\left|\tilde{M}_{\kappa}\left(\phi^{\prime}\right)\right|^{4 j} \leqq((2 j) !)^{4} b_{t}^{2 j}\left((\ln \kappa)^{m} \kappa^{-2+4 \varepsilon}\right)^{2 j} .
$$

(A.20) and (A.23) imply (A.19), which prove the lemma.

Remark. Analogous to (A.16) we also have the uniform bound

$$
E_{\phi}\left(e^{-\lambda \int_{0}^{t} d s M_{\kappa}\left(\phi_{s}\right)}\right) \leqq e^{C_{\phi}^{t}|\Lambda|}
$$

Acknowledgements. We thank Erhard Seiler for stimulating conversations on Stochastic quantization. G. J.-L. thanks S. Albeverio for a very useful discussion. P. K. M. thanks A. Martin, E. Seiler, W. Zimmerman and the members of the Werner Heisenberg Institut, Max Planck Institut für Physik, Munich and the Theory Division, CERN for their generous hospitality. G. J.-L. would like to express his gratitude to L. Streit, D. Elworthy, and A. Truman for their warm hospitality at the University of Bielefeld and the University of Swansea.

\section{References}

1. Glimm, J., Jaffe, A.: Quantum physics. Berlin, Heidelberg, New York: Springer 1981

2. Simon, B.: The $P(\phi)_{2}$ Euclidean (quantum) field theory. Princeton, NJ: Princeton University Press 1974

3. Nelson, E.: In: Constructive quantum field theory. Lecture Notes in Physics. Vol. 25, Velo, G., Wightman, A. (eds.). Berlin, Heidelberg, New York: Springer 1973

4. Glimm, J., Jaffe, A.: Quantum field models in statistical mechanics and field theory, Les Houches (1970), De Witt, C., Stora, R. (eds.). New York: Gordon and Breach 1971

5. Glimm, J.: Boson fields with nonlinear selfinteraction in two dimensions. Commun. Math. Phys. 8, 12-25 (1968)

6. Simon, B., Høegh-Krohn, R.: J. Funct. Anal. 9, 121-180 (1972)

7. Gross, L.: Am. J. Math. 97, 1061-1083 (1975)

8. Gross, L.: Harmonic analysis on Hilbert space. Memoirs AMS, Vol. 26

9. Gross, L.: Abstract Wiener spaces. In: Proceedings of the $5^{\text {th }}$ Berkeley Symposium on mathematical statistics and probability. Berkeley, CA: University of California Press 1968

10. Gross, L.: J. Funct. Anal. 10, 52-109 (1972)

11. Gelfand, I., Vilenkin, N.: Generalized functions. Vol. 4, New York: Academic Press 1964

12. Yosida, K.: Functional analysis. Berlin, Heidelberg, New York: Springer 1966

13. McKean, H.: Stochastic integrals. New York, London: Academic Press 1969

14. Stroock, D., Varadhan, S.R.S.: Multidimensional diffusion processes. Berlin, Heidelberg, New York: Springer 1979

15. Simon, B.: Functional integration and quantum physics. New York: Academic Press 1979

16. Friedman, A.: Stochastic differential equations, Vol. 1. New York: Academic Press 1975

17. Yor, M., Priouret, P.: Asterisque No. 22

18. Parisi, G., Wu, Yong-Shi: Sci. Sin. 24, 483 (1981)

19. Faris, W., Jona-Lasinio, G.: Large fluctuations for a nonlinear heat equation with noise. J. Phys. A 15, 3025 (1982)

20. Zwanzinger, D.: Covariant quantization of gauge fields without Gribov ambiguity. Nucl. Phys. B 192, 259 (1981)

Baulieu, L., Zwanziger, D.: Equivalence of stochastic quantization and the Faddeev-Popov ansatz. Nucl. Phys. B 193, 163 (1981) 
21. Floratos, E., Iliopoulos, J.: Equivalence of stochastic and canonical quantization in perturbation theory. Nucl. Phys. B 214, 392 (1983)

22. Floratos, E., Iliopoulos, J., Zwanziger, D.: A covariant ghost-free perturbation expansion for Yang-Mills theories. Nucl. Phys. B 241, 221 (1984)

23. Seiler, E.: 1984, Schladming Lectures. Max-Planck-Institut für Physik München, Preprint

24. Glauber, R.: Time-dependent statistics of the Ising model. J. Math. Phys. 4, 294-307 (1963)

25. Holley, R.: Free energy in a Markovian model of a lattice spin system. Commun. Math. Phys. 23, 87 (1971)

26. Holley, R., Stroock, D.: In one and two dimensions, every stationary measure for a stochastic Ising model is a Gibbs state. Commun. Math. Phys. 55, 37 (1977); Applications of the stochastic Ising model to the Gibbs states. Commun. Math. Phys. 48, 249 (1976); Z. Wahrscheinlichkeitstheorie Verw. Geb. 35, 87 (1976)

27. Holley, R., Stroock, D.: Ann. Probab. Vol. 4, No. 2, 195; J. Funct. Anal. 42, 29 (1981)

28. Faris, W.: J. Funct. Anal. 32, 342 (1979); Trans. Am. Math. Soc. 261, 579 (1980)

29. Wick, W.: Convergence to equilibrium of the stochastic Heisenberg model. Commun. Math. Phys. 81, 361 (1981)

30. Doss, H., Royer, G.: Z. Wahrscheinlichkeitstheorie Verw. Geb. 46, 107 (1978)

31. Dimock, J., Glimm, J.: Adv. Math. 12, 58 (1974)

Communicated by G. Mack

Received March 26, 1985 